\title{
Review
}

\section{Cosmological evolution of supermassive black holes in galactic centers unveiled by hard X-ray observations}

\author{
By Yoshihiro UedA ${ }^{* 1, \dagger}$ \\ (Communicated by Yasuo TANAKA, M.J.A.)
}

\begin{abstract}
We review the current understanding of the cosmological evolution of supermassive black holes in galactic centers elucidated by X-ray surveys of active galactic nuclei (AGNs). Hard X-ray observations at energies above $2 \mathrm{keV}$ are the most efficient and complete tools to find "obscured" AGNs, which are dominant populations among all AGNs. Combinations of surveys with various flux limits and survey area have enabled us to determine the space number density and obscuration properties of AGNs as a function of luminosity and redshift. The results have essentially solved the origin of the X-ray background in the energy band below $\sim 10 \mathrm{keV}$. The downsizing (or anti-hierarchical) evolution that more luminous AGNs have the space-density peak at higher redshifts has been discovered, challenging theories of galaxy and black hole formation. Finally, we summarize unresolved issues on AGN evolution and prospects for future X-ray missions.
\end{abstract}

Keywords: black holes, active galactic nuclei, cosmological evolution, X-rays

\section{Introduction}

1.1 SMBHs in galactic centers. Recent observations revealed that nearly all galaxies in the local universe contain supermassive black holes (SMBHs) in their centers. ${ }^{1}$ Their masses range from $\sim 10^{5}$ to $\sim 10^{10}$ times that of the Sun $\left(M_{\odot}\right)$. Remarkably, there is a strong correlation between the mass of the SMBH and that of the galactic bulge (or its luminosity, or stellar velocity-dispersion) over a wide range of the mass. ${ }^{1-7)}$ These facts indicate that the formation process of SMBHs and that of galaxies are strongly coupled to each other despite of the large difference in their physical sizes, $\sim \mathrm{AU}$ and $\sim \mathrm{kpc}$; in other words, SMBHs and galaxies must have "co-evolved" in the past.

SMBHs are responsible for one of the most energetic phenomena in the universe, called Active Galactic Nuclei (AGNs). Quasi-Stellar Objects (QSOs), many are observed at the distant universe, are a sub-class of AGNs with particularly high luminosities. When matter falls onto a SMBH

\footnotetext{
*1 Department of Astronomy, Kyoto University, Kyoto, Japan.

$\dagger$ Correspondence should be addressed: Department of Astronomy, Kyoto University, Kitashirakawa-Oiwakecho, Sakyoku, Kyoto 606-8502, Japan (e-mail: ueda@kusastro.kyoto-u.ac.jp).
}

("accretion"), it emits intense electromagnetic radiation over a wide range of wavelengths (from radio to gamma rays) by releasing its gravitational energy in the deep potential of the central SMBH. The bolometric luminosity $L$ is related to the mass accretion rate $\dot{m}$ as $L=\eta \dot{m} c^{2}$, where $\eta$ is the radiative efficiency and $c$ is the light velocity. In radiatively efficient accretion through optically thick disks, ${ }^{8)} \eta \sim 0.1$. AGN luminosities are huge, and can be as large as $10^{15}$ times that of the Sun. Also, AGNs often produce powerful outflow in forms of jets and disk winds. Thus, SMBHs are not merely "holes" that absorb everything as naively imagined. Through their strong radiation and kinetic power of outflow during AGN phase, SMBHs play key roles in regulating the star formation in galaxies and even affect their surroundings on a physical scale of galaxy clusters $(\sim \mathrm{Mpc}){ }^{9)}$

It is one of the most fundamental questions in modern astronomy how these SMBHs in galactic centers formed and co-evolved with their host galaxies in the history of the universe. As a SMBH accretes surrounding matter with a rest-frame mass of $m$, it gains its mass by $\frac{1-\eta}{\eta} m c^{2}$. Thus, AGNs are also the very process where the SMBHs "grow" by mass accretion. Hence, in order to understand the growth history of SMBH, we need to reveal the 
cosmological evolution of AGNs, i.e., "shining and growing" SMBHs.

$\mathrm{X}$-ray emission from an AGN is produced via Comptonization of seed photons by hot corona with a temperature of $\sim 100 \mathrm{keV}$ located in an innermost region of the accretion disk. The $\mathrm{SMBH}$ and accretion disk are surrounded by a larger $(\gtrsim 0.1 \mathrm{pc})$ structure consisting of gas and dust called a "torus". The torus is often assumed to have a donut-like shape, although its detailed geometry, physical conditions, and origins are not fully understood yet. Inside the torus hole, there are clouds that emit broad emission lines with velocities of $\gtrsim 1000 \mathrm{~km} \mathrm{~s}^{-1}$, called the broad line region. The narrow line region, which is responsible for producing emission lines with velocity widths of $\lesssim 1000 \mathrm{~km} \mathrm{~s}^{-1}$, is located above and below the torus hole.

According to the unified scheme of AGNs, ${ }^{10}$ the viewing angle with respect to the torus determines the observed characteristics of an AGN. The torus causes significant extinction to optical to ultraviolet lights emitted from the accretion disk and broad line region, and also photoelectric absorption of the X-ray continuum. When the line-of-sight toward the SMBH is unblocked by the torus (face-on view), one can observe the broad line region and unabsorbed X-ray continuum. This is called a "type-1" (or unobscured) AGN. When the torus blocks the line-of-sight (edgeon view), the broad lines cannot be observed in the optical spectra due to dust extinction and the X-ray spectrum is absorbed and becomes "hard" (i.e., flux at higher energies relative to that at low energies increases), as was demonstrated by Awaki et al. $(1991)^{11)}$ using the third Japanese X-ray satellite Ginga. Then, the object is classified as a "type-2" (or obscured) AGN. The basic idea of the unified scheme seems to successfully apply to most of local AGNs.

Many observations indicate that a major population of AGNs are obscured AGNs. Generally, obscured AGNs are more difficult to detect than unobscured ones because of dust extinction by the torus and/or the host galaxy. Among various AGN surveys in different wavelengths, X-ray observations, particularly those in "hard" X-rays above $2 \mathrm{keV}$, provide one of the most efficient and complete way to detect AGNs. The primary reason is their strong penetrating power against absorption. Figure 1 displays typical X-ray spectra of AGNs with various column densities of absorption. It is seen that as the column density increases, the flux at lower energies becomes more suppressed by photoelectric absorption, while the hard X-ray flux is less affected.
In this paper, we call AGNs whose absorptions are smaller and larger than $\log N_{\mathrm{H}}=24$ (corresponding to an opacity for Compton scattering of $\sim 1$ ) as "Compton-thin AGNs" and "Compton-thick AGNs", respectively. Another great advantage of using hard $\mathrm{X}$-rays is much less contamination from stars in the host galaxy compared with other wavelengths, making it possible to construct a clean AGN sample even at low luminosities.

1.2 The X-ray background (XRB). Strong Xray radiation is observed from all directions of the sky, known as the "X-ray background" (XRB) or the cosmic X-ray background (CXB). The XRB was discovered by Giacconi et al. (1962) with the famous rocket experiment when X-ray astronomy began. ${ }^{13)}$ Since then, to reveal the origin of the XRB, the oldest known cosmic background radiation in modern astronomy, has always been a prime theme of X-ray astronomy.

The XRB above a few $\mathrm{keV}$ is quite isotropic except for regions close to the Galactic plane, indicating that it has extragalactic origins. Its intensity is huge, amounting to 10 times larger than the integrated flux of all Galactic X-ray sources. The spectrum of the XRB has an intensity peak in $\nu F_{\nu}$ around $20-30 \mathrm{keV}$. Although the spectral shape can be approximated by thermal Bremsstrahlung emission with a temperature of $\sim 40 \mathrm{keV},{ }^{14}$ ) the presence of such a hot plasma filling the universe was later ruled out by the precise measurement of the Cosmic Microwave Background spectrum with the $C O B E$ experiment, $\left.{ }^{15}\right)$ which shows little distortion from blackbody radiation.

As we describe below, it is now established that the superposition of X-ray emission from the whole AGNs in the universe is observed as the XRB. Therefore, understanding the origin of the XRB is equivalent to revealing the cosmological evolution of AGNs that constitute the XRB. As the sensitivity of X-ray observatories increases, the fraction of the $\mathrm{XRB}$ resolved into discrete sources increases. To observe the X-ray sky with higher sensitivities in higher energy bands has been the advancement history of X-ray astronomy, including Japanese missions such as Ginga, ASCA, Suzaku, and ASTRO-H. Figure 2 plots the source number counts in the $2-$ $10 \mathrm{keV}$ band $(\log N-\log S$ relation) complied from various surveys.

\section{X-ray surveys of AGNs}

To reveal the cosmological evolution of AGNs over wide redshift and luminosity ranges, it is 


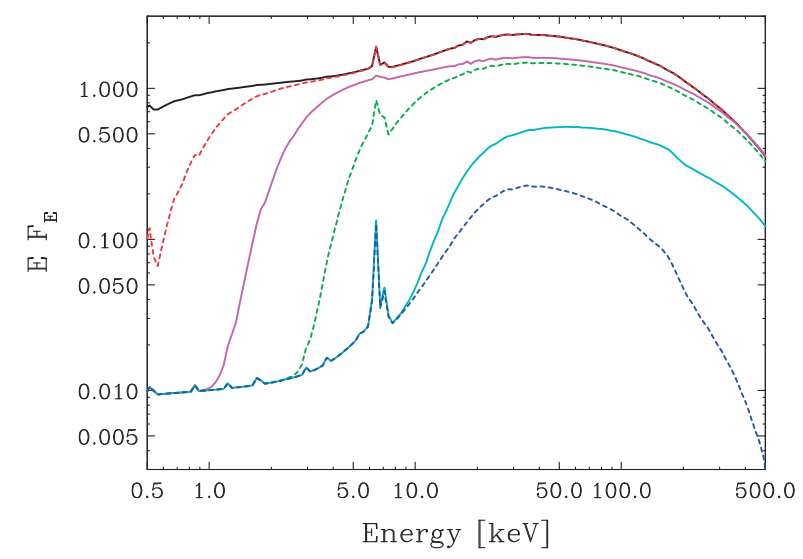

Fig. 1. Typical broad-band X-ray spectra ${ }^{12)}$ of AGNs with different absorptions (from top to bottom, $\log N_{\mathrm{H}}=20.5,21.5$, $22.5,23.5,24.5,25.5)$ in $E F_{E}\left(F_{E}\right.$ is energy flux at energy $\left.E\right)$. The scale is arbitrary.

necessary to perform a combination of unbiased X-ray surveys at different depths (sensitivity) and widths (area). To determine the redshift and type of each detected AGN, multi-wavelength follow-up observations, particularly optical spectroscopic ones, are required. In this section, we review the results of major X-ray surveys of AGNs at different sensitivity limits, focusing on those performed in energy bands above $2 \mathrm{keV}$.

2.1 Hard X-ray selected AGNs in the local universe. Understanding the statistical properties of AGNs in the local universe (i.e., at $z \simeq 0$ ) is particularly important because they give the reference of any cosmological evolution models. Nearby AGNs are bright, and hence detailed follow-up observations are possible once their locations are known. However, because their surface number density is low, one needs to make a wide-area survey, ideally an all-sky survey, to construct an unbiased AGN sample of sufficient size.

Following the first X-ray astronomy satellite Uhuru, launched in 1970, the HEAO-1 Observatory, launched in 1977, performed an all-sky X-ray survey. From the HEAO-1 A2 experiment, 30 AGNs were detected with a flux limit of $3.1 \times 10^{-11} \mathrm{erg} \mathrm{cm}^{-2} \mathrm{~s}^{-1}$ $(2-10 \mathrm{keV}) .{ }^{16)}$ This complete sample has long been the reference catalog of hard X-ray selected AGNs in the local universe. Later, Rossi X-ray Timing Explorer (RXTE) was launched in 1995, and its data taken during satellite reorientations (slew) were utilized to produce an all-sky X-ray catalog in the 3-20 keV band. ${ }^{20)}$ In 2009, the Monitor of All X-ray Image $(M A X I)^{21)}$ was launched to be installed on the

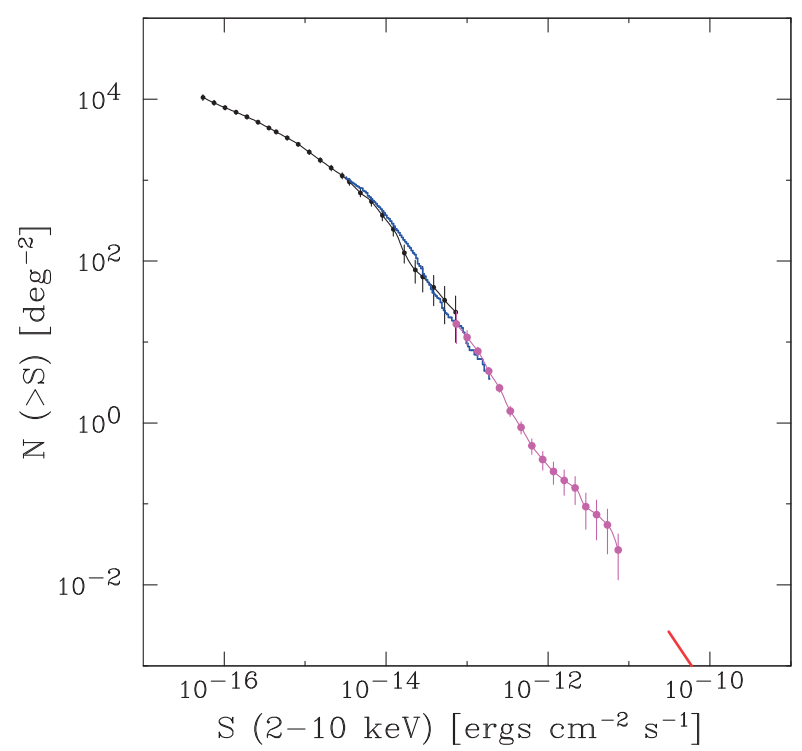

Fig. 2. Source number counts $(\log N-\log S$ relation) in the $2-$ $10 \mathrm{keV}$ band obtained from various surveys including HEAO-1 A2 survey $^{16)}$ (red), the ASCA Medium Sensitivity Survey ${ }^{17}$ ) (magenta), Subaru/XMM-Newton Deep Survey ${ }^{18)}$ (blue), and Chandra Deep Survey South ${ }^{19)}$ (black).

exposed facility of the Japanese experiment module "Kibo" in the international space station. Since then, $M A X I$ has been monitoring the entire sky to detect many transient sources. It also achieves the best sensitivity as an all-sky mission covering the 2$10 \mathrm{keV}$ band. The integrated data of MAXI have been used to produce unbiased X-ray source catalogs. Compiling the first 7 month data taken with MAXI/ Gas Slit Camera (GSC) at high Galactic latitudes $\left(|b|>10^{\circ}\right)$, Hiroi et al. $(2011)^{22)}$ detect 51 AGNs with a sensitivity of $1.5 \times 10^{-11} \mathrm{erg} \mathrm{cm}^{-2} \mathrm{~s}^{-1}(4-10 \mathrm{keV})$ with high identification completeness. The list of the brightest AGNs in the entire sky has substantially changed from that of the HEAO-1 A2 catalog due to time variability. The latest MAXI/GSC catalog ${ }^{23)}$ based on the 37 month data contains 500 sources. A majority of them are expected to be AGNs.

In energy bands above $10 \mathrm{keV}$, Swift/BAT and INTEGRAL satellite are performing all-sky surveys. ${ }^{24), 25)}$ These surveys are expected to provide the least biased AGN samples against absorption, except for heavily Compton thick AGNs with column densities larger than $\log N_{\mathrm{H}} \gtrsim 24.5$ (Fig. 1). Their results directly show that the dominant populations of local AGNs are obscured ones, in contrast to the fact that most AGNs found in the ROSAT all-sky survey $^{26)}$ at energies below $2 \mathrm{keV}$ are unobscured. The Japanese fifth X-ray astronomy Suzaku has 
performed follow-up observations of many AGNs detected with Swift/BAT. Suzaku has capability of simultaneously observing a wide energy band from $0.2 \mathrm{keV}$ to $600 \mathrm{keV}$. Suzaku discovered AGNs with very low scattered components in the X-ray spectra from the Swift/BAT sample, ${ }^{27)}$ many of which had not been detected previously. The results imply that these AGNs are deeply buried in geometrically thick tori. The observed fraction of Compton-thick AGNs in the whole sample is $\approx 6 \%$, implying that their intrinsic fraction is $\sim 30 \%$. ${ }^{12}$ )

2.2. Results from $\boldsymbol{A} \boldsymbol{S C A}$ surveys. $A S C A,{ }^{28)}$ the fourth Japanese X-ray mission launched in 1993, was the first X-ray astronomy satellite capable of imaging the hard X-ray sky in the $2-10 \mathrm{keV}$ band. $A S C A$ carried two types of focal plane detectors called the Gas Imaging Spectrometer (GIS) ${ }^{29)}$ and Solid-state Imaging Spectrometer (SIS), coupled to the X-Ray Telescope (XRT) ${ }^{30)}$ employing thin-foil optics sensitive up to $\sim 10 \mathrm{keV}$. It achieved $2-3$ orders of magnitude higher sensitivity in the $2-10 \mathrm{keV}$ band than those of any previous missions. To solve the origin of the hard XRB, a long standing puzzle in $\mathrm{X}$-ray astronomy, was one of the primary scientific goals to be addressed with $A S C A$.

Before ASCA, the Einstein (U.S.A.) and ROSAT (Germany) observatories, launched in 1978 and 1990, respectively, were also equipped with X-ray imaging mirrors, but they were only sensitive to soft X-rays below $2-3 \mathrm{keV}$. From deep surveys, they resolved $35 \%$ and $80 \%$ the soft XRB into discrete sources. ${ }^{31)-33)}$ From optical follow-up observations, the majority of the sources constituting the soft XRB were found to be type 1 (unobscured) AGNs. ${ }^{34)}$ However, the origin of the hard XRB above $2 \mathrm{keV}$ had never completely been explained by this population. This is because the X-ray spectra of type-1 AGNs are too soft to match the hard XRB: typical spectra of type-1 AGNs in the $2-10 \mathrm{keV}$ band are approximated by a power law with a photon index of $1.7-2.0,{ }^{35), 36)}$ while that of the XRB in the same band corresponds to a photon index of $\simeq 1.4^{14), 37), 38}$ This discrepancy was called the "spectral paradox", the biggest problem in understanding the XRB origin. To explain the shape of the XRB, the presence of other populations with much harder spectra than those of type-1 AGNs is necessary.

Using ASCA, Ueda et al. (1998) ${ }^{39)}$ performed the first imaging X-ray survey covering the $2-10 \mathrm{keV}$ band over a continuous region of $7 \mathrm{deg}^{2}$ in the Coma Berenices constellation (the ASCA Large Sky Survey, ALSS; Fig. 3). A specific analysis method was developed to extract the best capability of $A S C A$ by taking into account the complicated image responses. They detected total 107 sources, with a flux limit of $10^{-13} \mathrm{erg} \mathrm{cm}^{-2} \mathrm{~s}^{-1}$ in the 2-10 band, resolving $20-26 \%$ of the $2-10 \mathrm{keV} \mathrm{XRB}{ }^{40)}$ An important result is that they found evidence for the solution of the "spectral paradox" by detecting a significant fraction of sources with hard spectra. From optical spectroscopic followup observations, Akiyama et al. $(2000)^{41)}$ identified 30 AGNs detected above $2 \mathrm{keV}$. It is found that the population of hard sources are optical type- 2 AGNs (i.e., those without broad emission lines) at relatively low redshifts, $z<0.5$.

To further increase the sample size of the ALSS, a project called the $A S C A$ Medium Sensitivity Survey (AMSS) was conducted. The AMSS is a "serendipitous" source survey to systematically search for objects in the field-of-view of the GIS instrument other than the main target of each observation. The advantage is its very large survey area obtained by collecting all available observations performed during the life time of $A S C A$. Finally, Ueda et al. (2001, $2005)^{42), 43)}$ published the AMSS catalogs from the data taken between 1993-1996 and 1997-2000, which contain 1343 and 1190 X-ray sources, respectively. The $\log N-\log S$ relations in the $0.7-10 \mathrm{keV}$ band at these flux levels were determined with the best statistical accuracy, which solved the contradiction in source counts between soft $(<2 \mathrm{keV})$ and hard $(>2 \mathrm{keV})$ energy bands that had been reported previously. ${ }^{17)}$ The $A S C A$ results were basically confirmed by BерроSAX.44) Thanks to its large area, the AMSS has been a unique database to study X-ray populations at flux levels of $10^{-12}-10^{-13} \mathrm{erg} \mathrm{cm}^{-2} \mathrm{~s}^{-1}$ $(2-10 \mathrm{keV})$ up to the present. An optical follow-up observation program of a flux limited subsample of the AMSS was conducted. Using ground-based telescopes at University of Hawaii, Kitt-Peak observatory, Calar Alto observatory, and Cerro Tololo Inter-american Observatory, Akiyama et al. $(2003)^{45)}$ finally identified 86 AGNs with fluxes of $>3 \times$ $10^{-13} \mathrm{erg} \mathrm{cm}^{-2} \mathrm{~s}^{-1}(2-10 \mathrm{keV})$ with high completeness $(>98 \%)$.

2.3 Post $\boldsymbol{A} \boldsymbol{S C A}$ era. In 1999, Chandra and $X M M-N e w t o n$ were launched by NASA and ESA, respectively. With their excellent angular resolutions, these satellites achieved $>2$ orders of magnitude better sensitivities than that of $A S C A$. Soon after the launch, Chandra resolved $>75 \%$ of the hard XRB above $2 \mathrm{keV}$ into discrete sources. ${ }^{46)}$ Many deep surveys have been performed with Chandra and/or $X M M-N e w t o n$ on various fields, including the 
Chandra deep fields north ${ }^{47)}$ and south ${ }^{48)}$ (CDFN and CDFS), the Lockman Hole field, ${ }^{49}$ Groth Strip field ${ }^{50)}$ the Cosmic Evolution Survey (COSMOS) field ${ }^{51)}$ and the Subaru/XMM-Newton deep survey (SXDS) field. ${ }^{18)}$ The dominant populations of these faint X-ray sources turned out to be obscured AGNs, confirming the predictions by earlier works. A significant fraction of them have very faint optical counterparts for which optical spectroscopy is difficult. Multi-wavelength data are required to estimate their photometric redshifts. For a review of initial results obtained from Chandra and XMMNewton extragalactic surveys, we refer the reader to Brandt \& Hasinger (2005). ${ }^{52}$

The SXDS project was conducted by collaboration between the Subaru observatory and the $X M M$-Newton survey science center. It is one of the largest multi-wavelength surveys, covering from the radio, infrared, optical, and X-ray bands, with an unprecedented combination of depth and sky area over a continuous region of $>1 \mathrm{deg}^{2}$. The large area coverage is crucial to make an accurate measurement of the global properties of the universe without being affected by cosmic variance. Ueda et al. (2008) $)^{18)}$ published the X-ray source catalog of 1245 sources detected in the $0.3-10 \mathrm{keV}$ band with XMM-Newton/ European Photon Imaging Camera (EPIC) in the SXDS field. The sensitivity limit in the $2-10 \mathrm{keV}$ band is $3 \times 10^{-15} \mathrm{erg} \mathrm{cm}^{-2} \mathrm{~s}^{-1}$. The results of multiwavelength identification of the X-ray detected AGNs are reported by Akiyama et al. (2015, submitted). The X-ray image is displayed in Fig. 4. It is seen that a large fraction $(\sim 80 \%)$ of the XRB below $10 \mathrm{keV}$ is now clearly resolved into faint, individual sources, mostly AGNs.

\section{Cosmological evolution of AGNs}

3.1 X-ray luminosity function of AGNsdiscovery of "downsizing". The most important quantity that describes the cosmological evolution of AGNs is "luminosity function", which gives their co-moving space number density as a function of luminosity and redshift. As mentioned earlier, it is essential to make an unbiased AGN sample covering wide redshift and luminosity ranges, by combining multiple surveys with different flux limits. To accurately determine the luminosity function, the sample must be identified (i.e., the redshift of each AGN must be known) as completely as possible. Figure 5 gives the luminosity versus redshift plot of the hard X-ray selected AGN sample compiled by Ueda et al. (2003), ${ }^{53)}$ which consists of total 247
AGNs detected with HEAO-1, ASCA, and Chandra. The total completeness of redshift determination is very high $(100 \%, 97 \%$, and $93 \%$ for the $H E A O-1$, $A S C A$, and Chandra samples, respectively), making any uncertainties due to sample incompleteness least.

Using this sample, Ueda et al. (2003), ${ }^{53)}$ for the first time, quantitatively determine the hard X-ray luminosity function (XLF) of AGNs in the $2-10 \mathrm{keV}$ luminosity range of $10^{41.5}-10^{46.5} \mathrm{erg} \mathrm{s}^{-1}$ as a function of redshift up to $z=3$, including both type 1 and type 2 AGNs. The analysis is based on the maximum likelihood method fully correcting for observational biases with consideration of the X-ray spectrum of each source. The hard XLF, $d \Phi_{\mathrm{X}}\left(L_{\mathrm{X}}, z\right) / d \log L_{\mathrm{X}}$, represents the number density per unit co-moving volume per $\log L_{\mathrm{X}}$ as a function of $L_{\mathrm{X}}$ and $z$ in units of $\mathrm{Mpc}^{-3} \mathrm{dex}^{-1}$, where $L_{\mathrm{X}}$ is intrinsic (de-absorbed) $\mathrm{X}$-ray luminosity. Figure 6 plots the resultant hard XLF of Compton-thin AGNs in five redshift ranges. The lines show the best-fit model in each redshift bin, while the crosses represent the data. The shape of AGN XLFs is approximated by a double power-law function, whose slopes are different between above and below the break luminosity. As noticed, the evolution of the hard XLF is complicated, and its shape evolves with redshift. Ueda et al. (2003) $)^{53)}$ found that a luminosity-dependent density evolution (LDDE) model gives the best description of the results.

Figure 7 shows the co-moving space density of Compton-thin AGNs in three different luminosity ranges plotted against redshift. As noticed, the number density of AGNs rapidly increases with redshift, reaches a peak, and then slowly declines toward higher redshifts. The key finding here is that this peak redshift increases with AGN luminosity: more luminous AGNs are the most abundant at higher redshift than less luminous ones. This behavior indicates the "cosmic downsizing" or "antihierarchical evolution" of SMBHs: more massive black holes formed in earlier cosmic time than less massive ones ${ }^{* 1)}$. Although an LDDE formulation was firstly introduced to represent the XLF of AGNs detected with ROSAT in the soft X-ray band, ${ }^{54)}$ the luminosity dependence of the peak redshift was not found. This is most probably because soft X-ray surveys missed a large number of absorbed, lowluminosity AGNs (see Section 3.2).

*1) The term "downsizing" describes their global statistical properties, and should not be taken that the mass of an individual SMBH or galaxy decreases with cosmic time. 


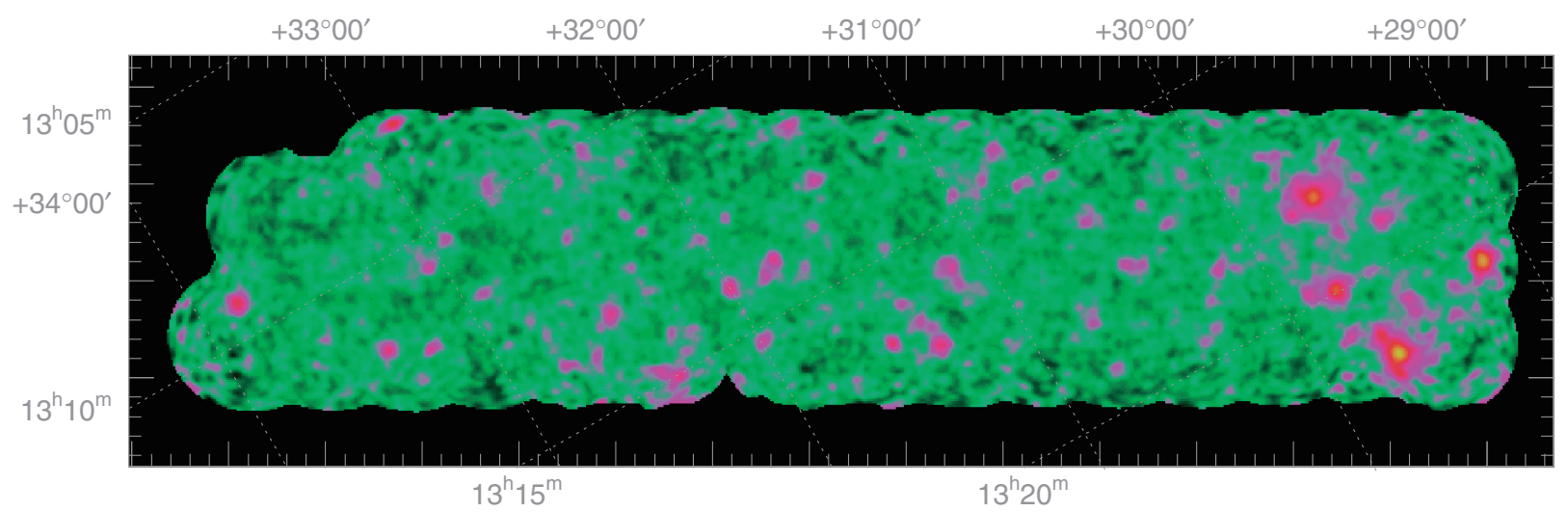

Fig. 3. X-ray image of the $A S C A$ Large Sky Survey field obtained with the GIS. ${ }^{29)}$ The image size is about $5^{\circ} \times 1^{\circ}$.

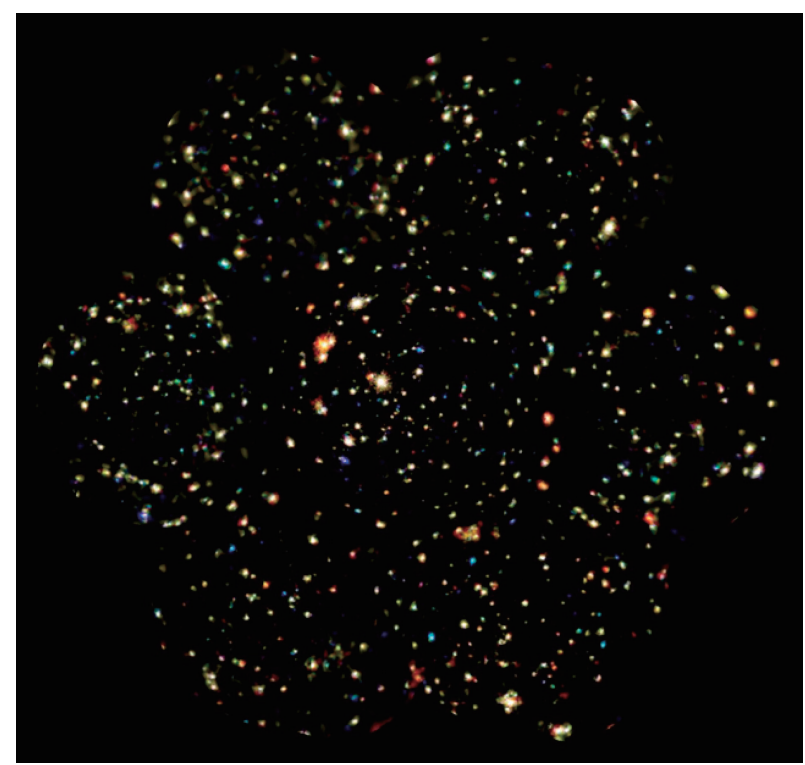

Fig. 4. X-ray image of the Subaru/XMM-Newton Deep Survey field, ${ }^{18)}$ consisting of 7 pointings with the EPIC, covering $1.14 \mathrm{deg}^{2}$ in total.

The discovery of the SMBH downsizing was striking because this trend is apparently opposite to a naive prediction from the standard structureformation theory of the universe based on cold dark matter models, where more massive dark matter halos formed more recently by merging from less massive ones. Note that, in this argument, there is an implicit assumption that the luminosity (hence the mass accretion rate) correlates with the black hole mass of the AGN. Recently, the assumption has been confirmed by measuring the black hole masses of Xray detected AGNs at different redshifts. ${ }^{55)}$ Possible origins of the cosmic downsizing are discussed in Section 4.3

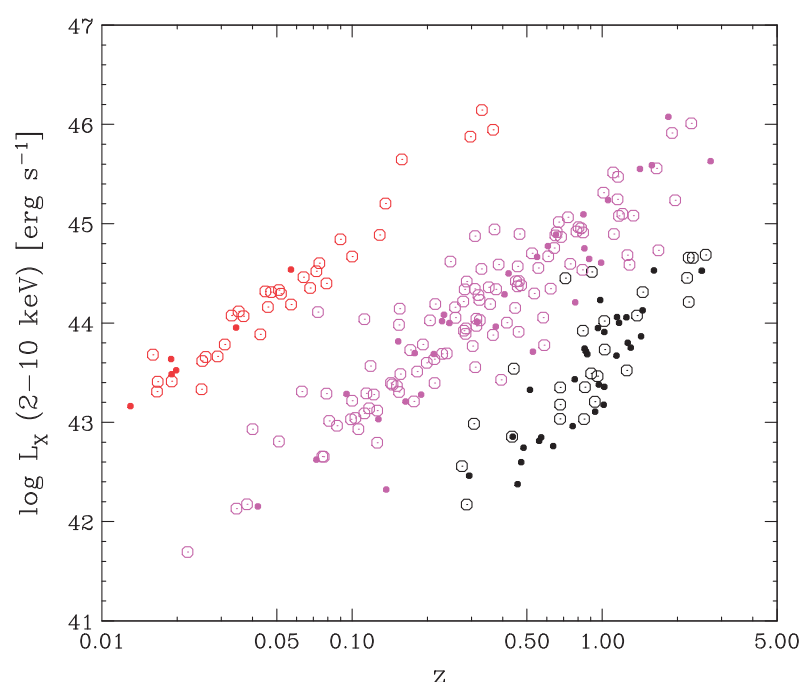

Fig. 5. Redshift versus luminosity plot of the sample used in Ueda et al. $(2003)^{53)}$ (red: HEAO-1 sample, magenta: ASCA sample, black: Chandra sample). The open and filled circles correspond to X-ray unabsorbed AGNs (with $\log N_{\mathrm{H}}<22$ ) and X-ray absorbed AGNs (with $\log N_{\mathrm{H}} \geq 22$ ) AGNs, respectively.

Since the publication of the first hard XLF of AGNs by Ueda et al. (2003), ${ }^{53)}$ more X-ray surveys and multi-wavelength identification observations have been performed, which provide larger samples included in later studies. ${ }^{56)-62)}$ One of the most up-todate hard XLFs has been published by Ueda et al. $(2014),{ }^{12)}$ based on currently the largest X-ray AGN sample, which consists of 4039 detections detected with MAXI, ASCA, Chandra, XMM-Newton, Swift, and ROSAT in the hard $(>2 \mathrm{keV})$ and/or soft $(<2 \mathrm{keV})$ bands. The results obtained from the SXDS are also included there. Figure 8 plots the luminosity versus redshift plot of the entire sample. The derived co-moving space density of Compton thin AGNs in 4 


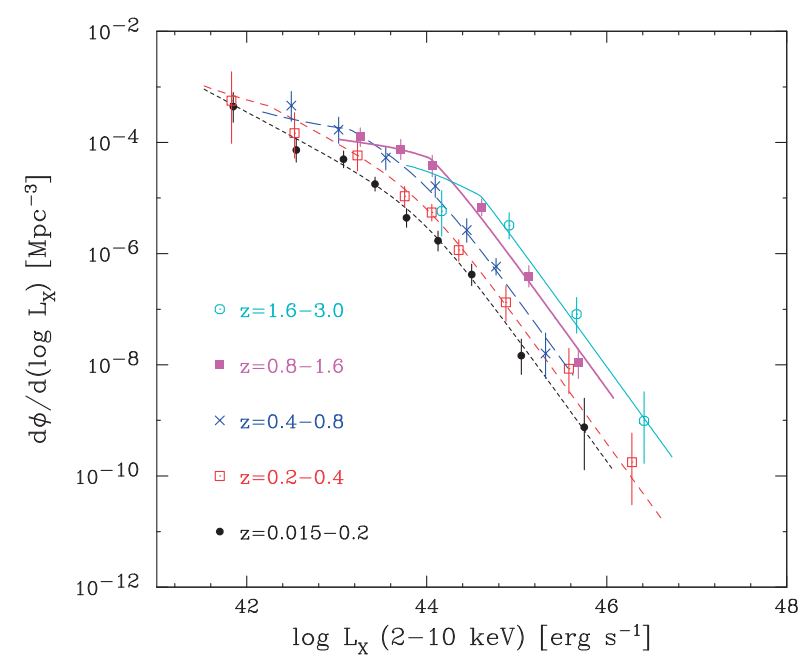

Fig. 6. Ueda et al. $(2003)^{53)}$ X-ray luminosity function of Compton-thin AGNs in five redshift bins, $z=0.015-0.2$ (shortdashed, black), 0.2-0.4 (medium-dashed, red), 0.4-0.8 (longdashed, blue), 0.8-1.6 (thick solid, magenta), and 1.6-3.0 (thin solid, cyan). The curves represent the best-fit LDDE models, and the data points are attached with their $1 \sigma$ statistical errors.

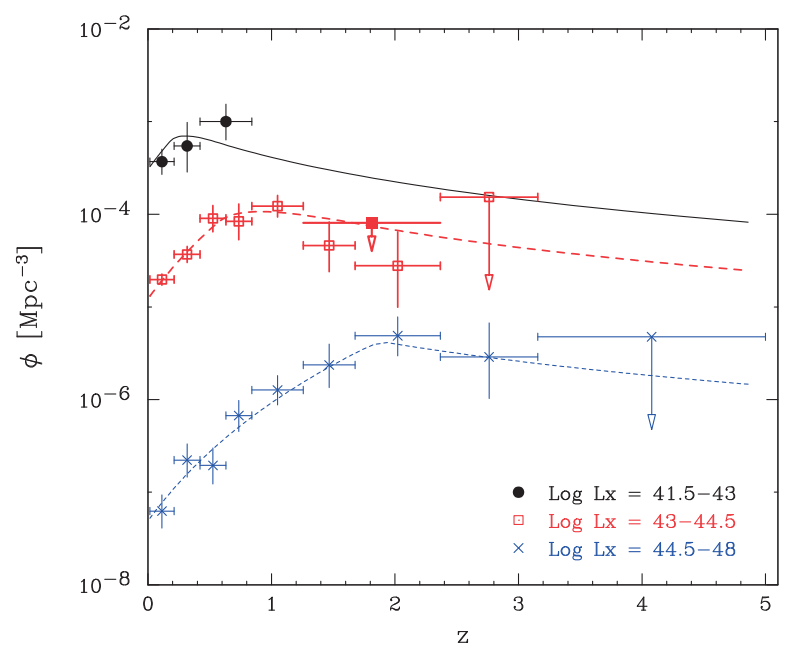

Fig. 7. Co-moving space density of Compton-thin AGNs derived by Ueda et al. $(2003)^{53)}$ as a function of redshift in three luminosity bins, $\log L_{\mathrm{X}}=41.5-43,43-44.5$, and $44.5-48$ from upper to lower. The curves are the best-fit models. The data points are attached with $1 \sigma$ errors. The long arrows denote $90 \%$ upper limits (statistical error only), while the short arrow (marked with the filled square) corresponds to the $90 \%$ upper limit by considering the maximum effect of the sample incompleteness.

luminosity bins is plotted in Fig. 9. The downsizing behavior is clearly confirmed by using the much larger sample than earlier ones. The decay of the AGN space-density above $z \gtrsim 3$ is noticed. Recent work utilizing the COSMOS sample has obtained a very similar result. ${ }^{63)}$

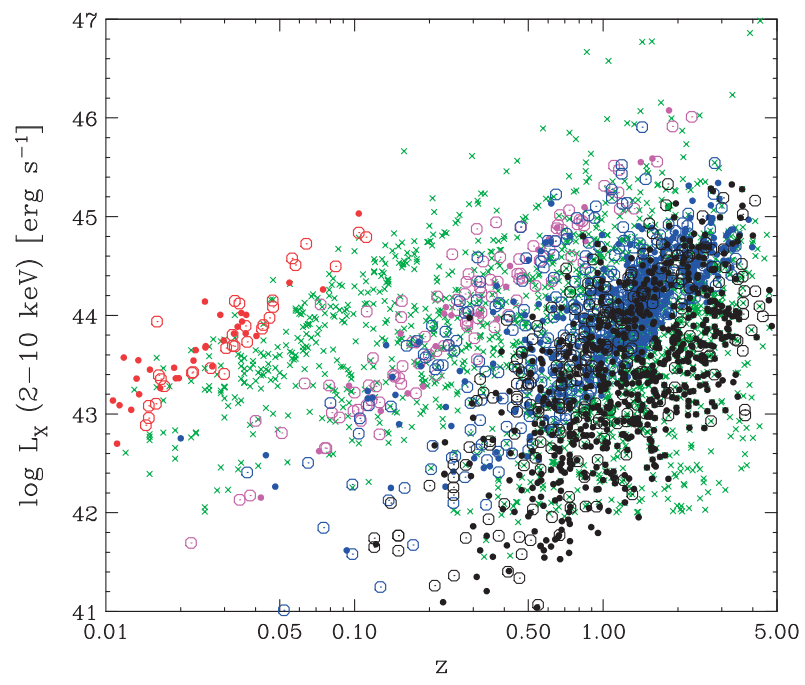

Fig. 8. Redshift versus luminosity plot of the sample used in Ueda et al. (2014). ${ }^{12)}$ The open and filled circles represent X-ray unabsorbed AGNs (with $\log N_{\mathrm{H}}<22$ ) and X-ray absorbed AGNs (with $\log N_{\mathrm{H}} \geq 22$ ), respectively, detected in the hard $(>2 \mathrm{keV}$ ) band (red: Swift/BAT sample, magenta: ASCA sample, blue: XMM-Newton sample, black: Chandra sample). Those detected in the soft $(0.5-2 \mathrm{keV})$ band are marked by crosses (green).

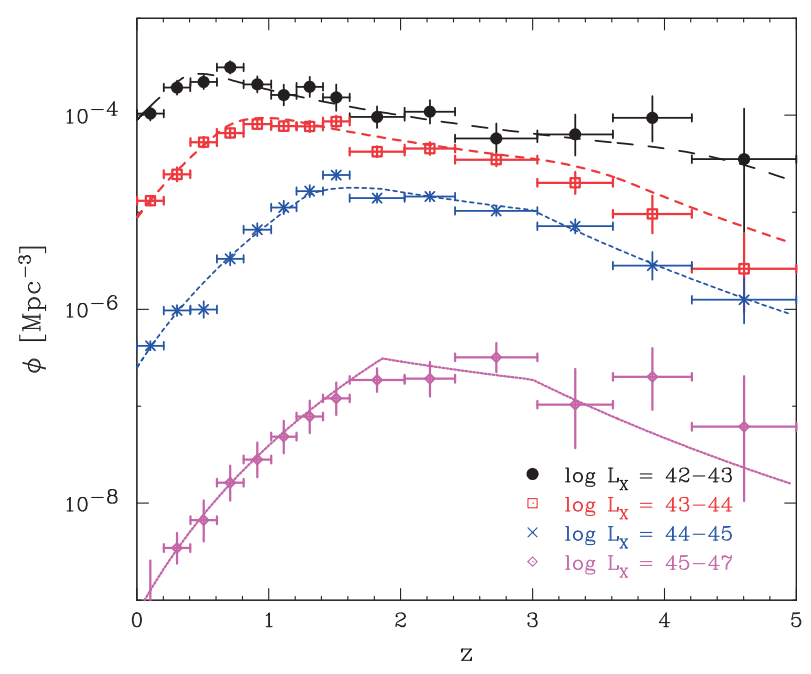

Fig. 9. Co-moving number density of Compton-thin AGNs derived by Ueda et al. $(2014)^{12)}$ as a function of redshift in four luminosity bins, $\log L_{\mathrm{X}}=42-43,43-44,44-45$, and 45-47, from upper to lower. The curves are the best-fit model, and the data points are attached with $1 \sigma$ errors.

3.2 Absorption properties of AGNs. Another important statistical property of AGNs is absorption distribution. This is related to the basic question whether or not the AGN unified scheme (Section 1.1) applies to all AGNs in the universe. For the quantitative evaluation, Ueda et al. $(2003)^{53)}$ intro- 
duce the "absorption function" (or " $N_{\mathrm{H}}$ function") of AGNs, a probability-distribution function for their absorption column-density. This carries information on the fraction of absorbed AGNs among all AGNs as a function of both luminosity and redshift.

Ueda et al. $(2003)^{53)}$ found that the absorbed AGN fraction with $\log N_{\mathrm{H}}>22$ decreases with the luminosity from their hard X-ray selected sample after correcting for selection biases, confirming the same trend previously reported from a local AGN sample. ${ }^{64)}$ It is consistent with deficiency of type 2 QSOs (i.e., type-2 luminous AGNs) in the sample of the ALSS. ${ }^{41)}$ The correlation has been confirmed by many authors using AGNs selected at different wavelengths. ${ }^{65)-67)}$ This fact rules out the simplest unified scheme where a torus of a fixed shape is surrounding the nucleus. Rather, the torus solidangle viewed from the SMBH decreases with the luminosity. The picture, referred as a modified unified-scheme ${ }^{53)}$ or a luminosity-dependent unified scheme, ${ }^{68)}$ also explains the anti-correlation between the AGN luminosity and the equivalent width of the narrow iron-K emission line known as X-ray Baldwin effect. ${ }^{69)}$ One plausible explanation is that the inner radius of the torus recedes as the luminosity increases as a result of dust sublimation, while the height of the torus has weaker dependence on luminosity. ${ }^{65)}$ The difference of the innermost torus structure between low and high luminosity AGNs is now observed by infrared interferometric observations of nearby AGNs. ${ }^{70)}$

By using larger samples, it has been also revealed that the absorbed AGN fraction depends on redshift, in the sense that there are relatively more absorbed AGNs at higher redshifts. ${ }^{12), 58), 71)-73)}$ Figure 10 plots the fraction of absorbed AGNs with $\log N_{\mathrm{H}}=22-24$ among all Compton-thin AGNs $\left(\log N_{\mathrm{H}} \leq 24\right)$ against luminosity in two redshift bins $(0.1<z<1$ and $1<z<3) .{ }^{12)}$ As noticed, the absorbed AGN fraction increases with redshift by keeping the anti-correlation with luminosity. This fact means that the unified scheme must be also modified by including evolution effects. It may suggest that the averaged covering factor of tori is larger at higher redshifts. Alternatively, interstellar absorption in the host galaxy on a larger spatial scale than the torus may play a role. In fact, the host galaxies of obscured AGNs at $z \approx 1.5-3$ exhibit much larger star forming rates than those of unobscured AGNs at the same redshift, ${ }^{74)}$ suggesting that their evolutional stages are quite different. Thus, while the unified scheme explains many observational aspects of AGNs in the local universe, it cannot be simply applied to all AGN phenomena in the universe.

\section{Implications}

4.1 Population synthesis of the X-ray background. On the basis of various hard X-ray surveys performed at different flux limits, the hard XLF and absorption function of AGNs have been determined as a function of redshift and luminosity. In other words, the cosmological evolution of AGNs that constitute a major part of the XRB below $\approx 10 \mathrm{keV}$, including the dominant type- 2 populations, is now revealed. This is regarded as an unique achievement by hard X-ray observations, which enable us to detect absorbed AGNs with the least biases. The formulations of these functions over the luminosity and redshift ranges of $\log L_{\mathrm{X}}=42-47$ and $z=0-5$ are presented in Ueda et al. (2014). ${ }^{12)}$

The combination of the XLF and absorption functions enables us to construct a so-called "population synthesis model" of the XRB, ${ }^{53), 71), 75)-77)}$ where the contributions to the XRB spectrum from AGNs with different luminosities, redshifts, and absorptions are quantitatively described. Since the $\mathrm{XRB}$ below $\approx 10 \mathrm{keV}$ has been resolved into AGNs that are used to determine the two functions, we can make "consistency-check" if the integrated spectrum of the whole AGNs reproduces the XRB spectrum in that energy band. In addition, it is possible to predict their integrated flux above $10 \mathrm{keV}$, where the XRB is not yet directly resolved, by assuming broad band spectra of AGNs.

Ueda et al. $(2014)^{12)}$ present the most up-todate XRB synthesis model based on their XLF and absorption function of AGNs. They consider (1) the evolution of absorption function, (2) broad band $\mathrm{X}$-ray spectra including reflection components from the torus based on the luminosity and redshift dependent unified-scheme, and (3) contribution of Compton-thick AGNs (i.e., those with $\log N_{\mathrm{H}}>24$ ). In the analysis, the number ratio of Compton-thick AGNs to Compton-thin, absorbed ones $\left(\log N_{\mathrm{H}}=22-\right.$ 24 ) is assumed to be constant regardless of luminosity and redshift. Figure 11 compares the broad-band $\mathrm{XRB}$ spectra measured by various observatories and the integrated spectrum from all AGNs with $\log L_{\mathrm{X}}=41-47$ at $z=0.002-5.0$ based on this model. The second top curve represents the total contribution only from Compton-thin AGNs, which cannot fully account for the peak intensity of the hard XRB around $\sim 20 \mathrm{keV}$. The summed contribution from both Compton-thin and Compton-thick AGNs is 
given by the top curve, which is well consistent with the observed XRB; in other words, the presence of significant number of Compton-thick AGNs is indeed required to explain the "missing flux" in the hard XRB above $10 \mathrm{keV}$. It is found that AGNs with $\log L_{\mathrm{X}} \approx$ 43.8 at $z \approx 1.1$ make the largest contribution to the $\mathrm{XRB}$ in the $2-10 \mathrm{keV}$ band per unit $\log L_{\mathrm{X}}$ and $z$.

4.2 Growth history of SMBHs. As mentioned in Section 1.1, a SMBH gains its mass from accreted matter, and therefore the luminosity of an AGN represents the mass growth rate of each $\mathrm{SMBH}$ through the radiative efficiency $\eta$. Thus, it is possible to reveal the global growth history of SMBHs by accretion once the AGN luminosity function is determined as a function of redshift. This argument was pointed out by Soltan (1982). ${ }^{79)}$ The total mass density of SMBHs (including non-active SMBHs), $\rho(z)$, can be calculated by integrating the massaccretion-rate density, $\dot{\rho}(z)$, over cosmic time, as

$$
\rho(z)=\int_{z}^{\infty} \dot{\rho}(z) d z \frac{d t}{d z}
$$

where

$$
\dot{\rho}(z)=\frac{1-\bar{\eta}}{\bar{\eta} c^{2}} \int L \frac{d \Phi_{\mathrm{bol}}(L, z)}{d \log L} d \log L .
$$

Here $\Phi_{\text {bol }}(L, z)$ is a bolometric luminosity function (defined for the luminosity $L$ integrated over all wavelengths) and $\bar{\eta}$ represents an averaged radiation efficiency that is assumed not to depend on $z$ or $L$ in this formula. One can then compare this result with the SMBH mass density at $z=0$ independently measured from the relation between SMBH mass and bulge mass. This leads us to constrain $\bar{\eta}$, which reflects the accretion modes in AGNs (e.g., standard $\operatorname{disk}^{8)}$ or radiatively inefficient accretion flow ${ }^{80)}$ ) and the spin parameters of SMBHs.

The discussion based on the latest hard XLF is presented in Ueda et al. (2014). ${ }^{12)}$ The bolometric luminosity function of all AGNs (including Comptonthin and Compton-thick AGNs) is derived from the hard XLF by assuming luminosity-dependent bolometric correction factors and their scatter. ${ }^{81)}$ In their model, it is found that $\approx 74 \%(\approx 37 \%)$ of the total energy emitted by whole AGNs in the history of universe was produced by obscured accretion with $\log N_{\mathrm{H}}=22-26\left(\log N_{\mathrm{H}}=24-26\right)$. Figures 12 and 13 respectively plot $\dot{\rho}(z)$ and $\rho(z)$ by adopting $\bar{\eta}=0.04$, in luminosity ranges of $\log L=43-48$ and $\log L=$ 46-48. As expected from the downsizing evolution, the contribution from less luminous AGNs becomes more significant at lower redshifts.
The value of $\rho(z=0)$ is in a good agreement with the observed local SMBH mass density, ${ }^{12}$ ) $\rho^{\text {obs }}(z=0) \approx 1 \times 10^{6} M_{\odot} \mathrm{Mpc}^{-3}$ calculated by using the recently updated SMBH-mass versus bulge-mass relation. ${ }^{7)}$ The radiative efficiency of $\eta \approx 0.04$ does not require extremely radiatively inefficient accretion flow as a predominant accretion mode of AGNs.

Furthermore, it is also possible to trace the mass function of SMBHs (including non-active $\mathrm{SMBHs}$ ) from the AGN luminosity function by neglecting merging of SMBHs. ${ }^{82)-84)}$ In this analysis, one needs to introduce the Eddington ratio $\lambda$, the bolometric luminosity divided by the Eddington luminosity, given as $L_{\mathrm{Edd}}=1.25 \times 10^{38}\left(M / M_{\odot}\right) \mathrm{erg} \mathrm{s}^{-1}$ for a black hole mass of $M$. By comparing with the observed mass functions, it is argued that the radiative efficiency should decrease with luminosity. ${ }^{12), 85)}$ The inferred high radiative efficiencies at large $M$ suggests that these SMBHs have high spin parameters (i.e., rapidly rotating). In order to obtain robust conclusions, it is critical to observationally determine the Eddington-ratio distribution function and black hole mass function of AGNs, as a function of redshift, by covering a wide luminosity range.

To compare the growth history of SMBHs and that of galaxies, we overplot the star-forming-rate density and steller-mass density in Figs. 12 and 13, respectively, based on a recent compilation by Madau and Dickinson (2015). ${ }^{86}$ ) They are rescaled by a factor of 0.002 . The overall similarity between the mass accretion rate (or SMBH mass density) and star forming rate (or stellar mass density) supports the global "co-evolution" scenario, as previously suggested. ${ }^{87)}$ A small discrepancy is noticed at $z>3$ (corresponding to the cosmic age of $<2.2 \mathrm{Gyr}$ ). This could be explained if the peak epoch of AGN activity in an individual galaxy is, on average, delayed by a timescale of Gyr from that of starburst.

4.3 Origin of downsizing. The most remarkable finding from the hard X-ray AGN surveys is the downsizing or anti-hierarchical evolution of SMBHs, indicating that present-day more massive black holes formed earlier in cosmic time than less massive ones. Similar trends have been observed in star formation of galaxies; it was firstly suggested by Cowie et al. $(1996)^{88)}$ and many recent observations have established this picture (e.g., Kodama et al. (2004); ${ }^{89)}$ see Fontanot et al. $(2006)^{90)}$ and references therein). If the co-evolution of SMBHs and galaxies is almost simultaneous, this implies that same origins may work for the downsizing seen in both AGNs and star formation. 


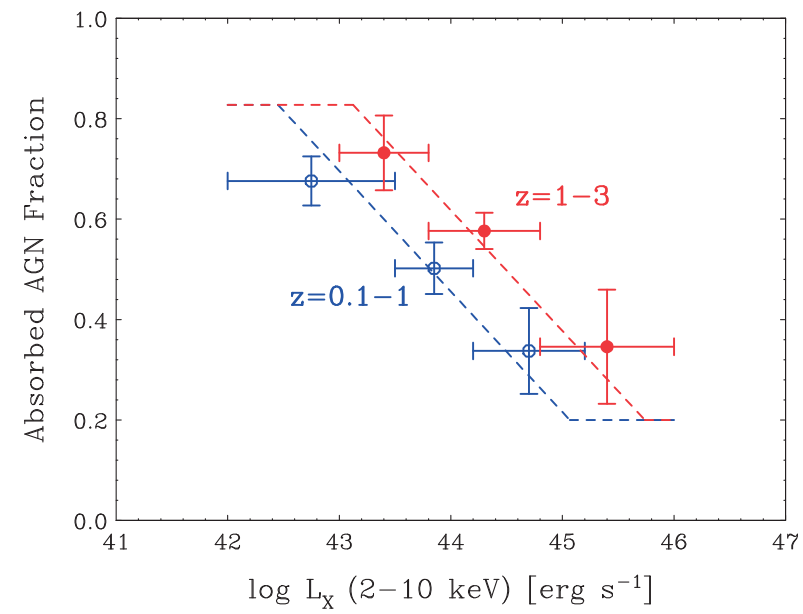

Fig. 10. Fraction of absorbed AGNs $\left(\log N_{\mathrm{H}}=22-24\right)$ among all Compton-thin AGNs $\left(\log N_{\mathrm{H}}<24\right)$ plotted against luminosity at $z=0.1-1$ (blue) and $z=1-3$ (red) as determined from the Swift/ BAT, AMSS, and SXDS samples. The dashed lines correspond to the best-fit models by Ueda et al. (2014). ${ }^{12)}$

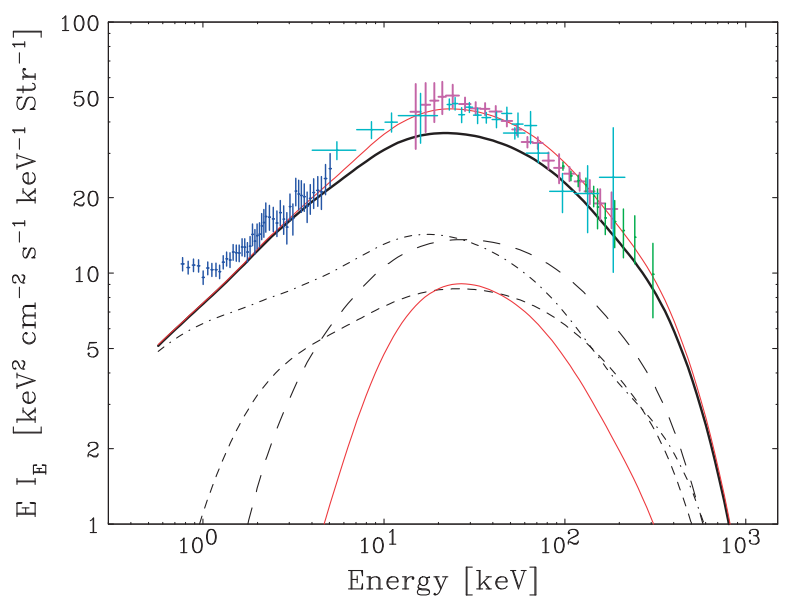

Fig. 11. Integrated spectrum from all AGNs at $z<5$ based on the population synthesis model by Ueda et al. $(2014)^{12)}$ (upper solid curve, red). Middle solid curve (black): the integrated spectrum of Compton-thin AGNs $\left(\log N_{\mathrm{H}} \leq 24\right)$. Lower solid curve (red): that of Compton-thick AGNs $\left(\log N_{\mathrm{H}}=24-26\right)$. Long-dashed curve (black): that of AGNs with $\log N_{\mathrm{H}}=23-24$. Short-dashed curve (black): that of AGNs with $\log N_{\mathrm{H}}=22-23$. Dot-dashed curve (black): that of AGNs with $\log N_{\mathrm{H}}<22$. The data points are the XRB spectra observed with various missions. ${ }^{78)}$

The downsizing evolution does not conflict with the standard "bottom-up" structure-formation scenario in cold dark matter models, which has now been well accepted. This is because the SMBH growth seen as AGNs does not necessarily have to follow the process of their "assembly". For instance, the observed decrease of the space density of

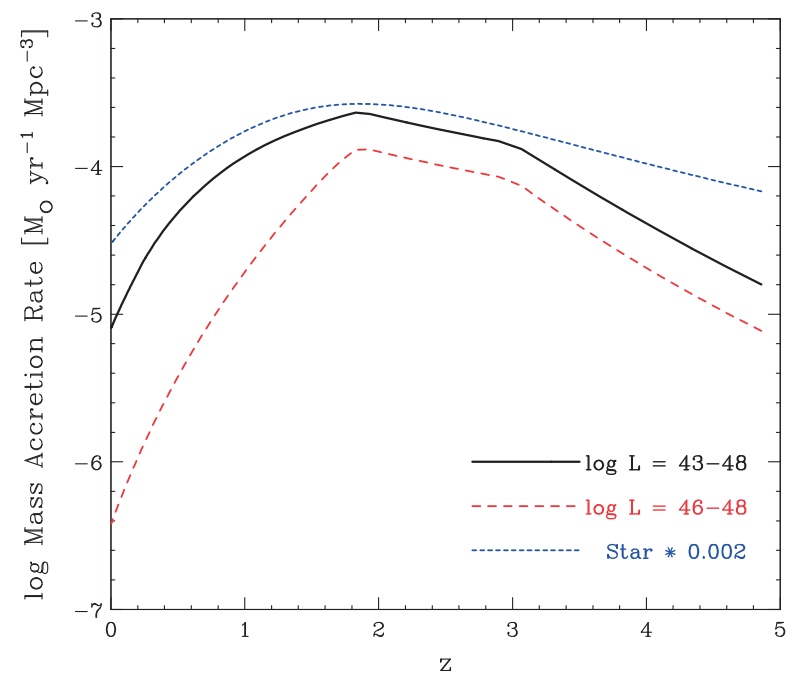

Fig. 12. Co-moving mass-accretion-rate density onto SMBHs as a function of redshift by assuming a radiation efficiency of $\bar{\eta}=0.04$. The solid (black) and long-dashed (red) curves are calculated from AGNs with bolometric luminosities of $\log L=$ 43-48 and $\log L=46-48$, respectively. The short-dashed (blue) curve represents the co-moving star-forming-rate density ${ }^{86)}$ rescaled by 0.002 .

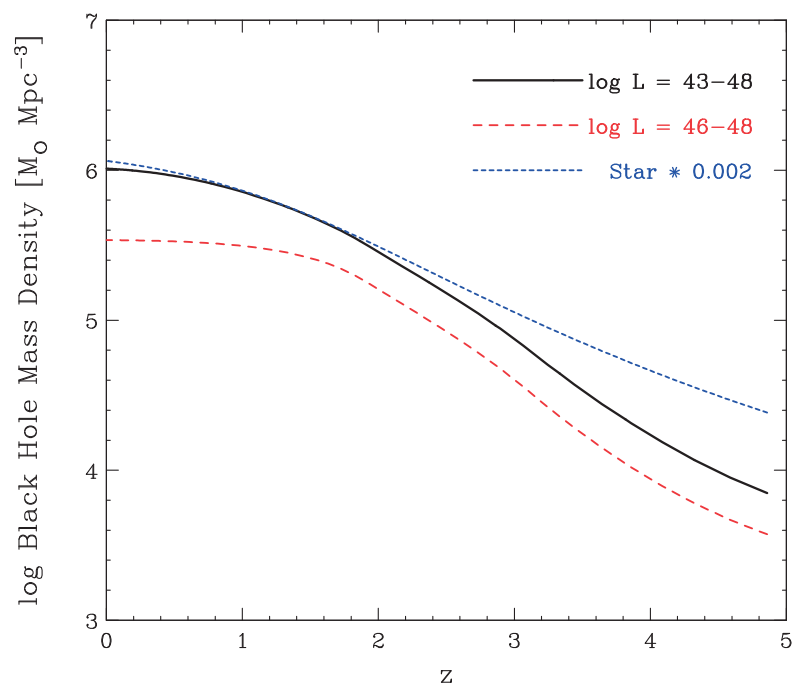

Fig. 13. Growth curve of SMBHs by accretion, where the comoving mass density of SMBHs is plotted against redshift. A radiation efficiency of $\bar{\eta}=0.04$ is assumed. The solid (black) and long-dashed (red) curves represent the contribution from AGNs with bolometric luminosities of $\log L=43-48$ and $\log L=46-48$, respectively. The short-dashed (blue) curve plots the co-moving stellar mass density ${ }^{86)}$ rescaled by 0.002 .

luminous AGNs at $z<2$ means that merging of massive dark matter halos does not lead to rapid growth of SMBHs at low redshifts. We need to understand baryon-specific physics other than pure gravity governing the dynamics of dark matter halos. 
After the discovery, understanding the downsizing evolution has been a major challenge to theoretical models of galaxy and SMBH formation, both in semi-analytic models and cosmological hydrodynamic simulations. Earlier simple theoretical models ${ }^{91), 92)}$ did not predict any downsizing behaviors. Recent works ${ }^{93)-97)}$ have started to reproduce the downsizing trend to fit the observations, although no consensus has been established yet on the origins. An important effect may be that the cooling of gas necessary to "fuel" the AGN is suppressed in very massive halos at low redshifts, due to AGN feedback in the so-called "radio mode" or "kinetic mode", which takes place when the accretion rate onto the $\mathrm{SMBH}$ is sufficiently low. $\left.{ }^{9}\right)$ Combined with depletion of cold gas by star formation and an increase of accretion time-scale with decreasing redshift, this could cause a quicker decrease of the space density of luminous AGNs than that of less luminous AGNs. ${ }^{97}$ )

Likely scenarios are that there are at least two mechanisms for AGN fueling, major mergers and other processes such as disk instabilities ${ }^{95)}$ and hothalo accretion. ${ }^{93), 94)}$ This picture is also consistent with the observational results on the morphology of AGN hosts; a significant fraction are disk-dominated galaxies (i.e., without evidence for past major mergers). ${ }^{98), 99)}$ Most of those models, however, overpredict the space density of low luminosity AGNs at high redshifts $(z>1-2)$ compared with the observations. ${ }^{63)}$ The reason is yet unclear; some important physics would still be missed. Alternatively, if there are a large number of heavily Compton-thick AGNs with low luminosities at $z>1$ that are missing in current surveys, it could solve the discrepancy.

\section{Prospects for future observations}

The hard X-ray surveys detected AGNs of various luminosities and redshifts, covering 6 orders of magnitude in flux. Type-2 (absorbed) AGNs are found to be the major population, which were easily missed in other wavelengths. As a result, a global picture of cosmological evolution of AGNs, in particular the cosmic downsizing, has been revealed. At the same time, most of the XRB origin below $\approx 10 \mathrm{keV}$ is now quantitatively explained by superposition of these AGNs. Then, can we say that the whole picture of SMBH evolution in galactic centers has been completely solved? The answer is definitely "No". In this section, we focus on two important issues that must be pursued by future X-ray observations.
5.1 Evolution of AGN XLF at high redshifts. The evolution of SMBHs in the dawn of the universe is a critical issue in order to understand their initial formation mechanism. The most distant AGN (QSO) currently known is at $z=7.085$, whose black hole mass is estimated to be $2 \times 10^{9} M_{\odot} \cdot{ }^{100)}$ It is a big problem how such very massive systems formed in such a short time (within $7.7 \times 10^{8} \mathrm{yr}$ ) after the Big Bang. If this QSO had grown solely by accretion at the Eddington-limited accretion rate from a "seed" black hole of $100 M_{\odot}$, the required time would have been $7.6 \times 10^{8} \mathrm{yr}$ by assuming a radiative efficiency of $\eta=0.1$. Thus, high-redshift AGN surveys are also related to the question how the first "seed" black holes formed.

Because of their small space density, a wide-area survey is required to unbiasedly detect AGNs at very high redshifts. In fact, most of known distant QSOs at $z>5$ were firstly found by wide area optical or near-infrared surveys, such as the Sloan Digital Sky Survey. Future and on-going wide-area optical to near-infrared surveys, like those utilizing the Hyper Suprime-Cam on Subaru telescope will largely increase the sample.

However, the hard X-ray surveys indicate that a major population of AGNs are obscured ones, whose fraction increases with decreasing luminosity. Thus, observations by hard X-rays (at source frame) are indispensable to understand the whole story. While downsizing evolution of SMBHs on the cosmological time scale $(z \sim 3$ to $z=0)$ has been found, the inverse trend (i.e., "up-sizing", where the ratio of the space density of less luminous AGNs to more luminous ones increases with redshift) may be expected at higher redshifts. ${ }^{12)}$ To detect high redshift AGNs at $z>5$ covering a low luminosity range, we need to perform "very wide and very deep" X-ray surveys, which are practically impossible by current X-ray observatories. This is a prime scientific goal to be addressed by Athena, ${ }^{* 2)}$ the next large mission of ESA. Athena will bring a large sample of high $z$ AGNs at $z=5-10$, giving us the first unbiased view of SMBH formation process in the early universe.

5.2 Evolution of Compton-thick AGNs. How many heavily obscured (Compton-thick) AGNs exist in the universe and what are their contributions to the total growth of SMBHs are key questions to establish a complete picture of AGN evolution. Most of present X-ray surveys were performed in energy bands below $10 \mathrm{keV}$, which are not sufficient to catch

\footnotetext{
*2) http://www.the-athena-x-ray-observatory.eu/.
} 
the primary emission component from Comptonthick AGNs (Fig. 1). Although all-sky hard X-ray surveys above $\sim 10 \mathrm{keV}$ have started to find a part of this population in the local universe, the cosmological evolution of their XLF is essentially unknown.

In the population synthesis model by Ueda et al. (2014), ${ }^{12)}$ Compton-thick AGNs follow the same evolution as Compton-thin AGNs, by implicitly assuming that they are an extreme case of less absorbed, Compton-thin AGNs. The spectrum of the XRB gives a boundary condition; in this model, the number ratio of Compton-thick AGNs to Comptonthin absorbed AGNs is constrained to be $50 \%-160 \%$ within an uncertainty in the XRB intensity in the 20-50 keV band. We must keep it in mind, however, that any constraints for the number density of Compton-thick AGNs in population synthesis models strongly depend on basic assumptions, (1) the (unknown) evolution of the XLF of Compton-thick AGNs, and (2) detailed shape of the broad band spectra, in particular, the strength of reflection components that produce spectral hump at $\sim 30 \mathrm{keV} .{ }^{101}$ )

It is not clear whether or not Compton-thick and Compton-thin AGNs are intrinsically the same population. Theories predict that galaxies may contain Compton-thick AGNs shortly after major mergers, which are surrounded by huge amount of dust produced by the merger-driven starburst activities. ${ }^{102), 103)}$ These Compton-thick AGNs may be in a rapidly growing phase of $\mathrm{SMBHs}$, and hence are key objects to understand the mechanism of the galaxy-SMBH co-evolution. Ultra-luminous infrared galaxies (ULIRGs) may represent one of such populations, which are found to ubiquitously contain such obscured AGNs. ${ }^{104)}$

To directly unveil the cosmological evolution of Compton-thick AGNs, and their contribution to the hard XRB, the only way is to perform hard X-ray surveys in the energy band above $10 \mathrm{keV}$ with various depths and widths, as done in the past $\sim 50$ years at energies below $10 \mathrm{keV}$. The NuSTAR and ASTRO-H missions will resolve $\sim 30 \%$ of the hard XRB into individual AGNs, providing us with new insights on Compton thick populations. An initial result from the NUSTAR extragalactic survey based on ten $\mathrm{AGNs}^{105)}$ is consistent with the prediction by Ueda et al. (2014). ${ }^{12)}$ Eventually, however, much deeper hard X-ray surveys, with $>10$ times better sensitivities than $N u S T A R$ and $A S T R O-H$, are indispensable, in order to detect AGNs that compromise $\sim 80 \%$ of the hard XRB. A standard XRB model predicts that the fraction of Compton-thick AGNs

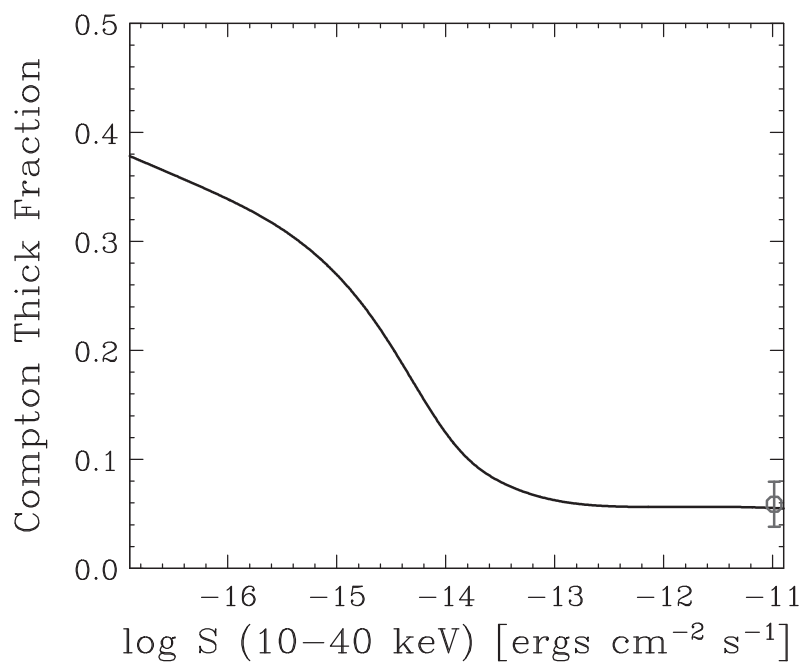

Fig. 14. Fraction of Compton-thick AGNs $\left(\log N_{\mathrm{H}}=24-26\right)$ in the total AGNs $\left(\log N_{\mathrm{H}} \leq 26\right)$ given as a function of $10-40 \mathrm{keV}$ flux predicted from the Ueda et al. $(2014)^{12)}$ model. The data point at the brightest flux is obtained from the Swift/BAT 9month survey.

drastically increases around this flux limit (Fig. 14). Then, it also becomes possible to constrain the XLF of Compton-thick AGNs with $\log L_{\mathrm{X}}>42$ by covering the redshift corresponding to the number-density peak inferred from the XLF of Compton-thin AGNs.

Even hard X-ray surveys above $10 \mathrm{keV}$ are subject to detection biases against "heavily Comptonthick" AGNs with $\log N_{\mathrm{H}}>25$, whose hard X-ray flux of the primary emission is significantly suppressed by repeated Compton scattering. Surveys at restframe far- to mid-infrared wavelengths, which have also little biases against obscuration, could potentially "detect" such populations although it is not trivial how to discriminate AGN and star-formation activities. Thus, X-ray and infrared bands are quite complementary to each other in studying heavily obscured AGNs, indicating the importance of multiwavelength approach.

\section{Conclusion}

We have reviewed the history of X-ray surveys of AGNs, growing SMBHs in galactic centers, with particular emphasis on observations at energies above $2 \mathrm{keV}$, the best bandpass to find them with least biases and contamination. Japanese X-ray astronomy observatories, including Ginga, ASCA, Suzaku, and $M A X I$, have made crucial contribution to this subject, along with many follow-up observations, which demonstrate the power of multiwavelength astronomy. 
These observations have made it possible to trace the cosmological evolution of a majority of AGNs in the universe, including the dominant, obscured AGN populations. As the consequence, the cosmic "downsizing" of SMBHs has been revealed, where more luminous AGNs show their numberdensity peak in an earlier epoch than less luminous ones. The apparently "anti-hierarchical" evolution has challenged a naive expectation from the standard structure-formation theory of the universe, requiring detailed understanding of baryon-specific physics other than gravity in the galaxy-SMBH co-evolution. The fraction of obscured AGNs is larger at higher luminosities and at higher redshifts. The origin of the XRB is now quantitatively explained by superposition of X-ray emitting AGNs in a standard population synthesis model. The evolution of the AGN luminosity density reveals the accretion history of the universe, and accounts for the present-day mass density of SMBHs mostly by radiatively efficient accretion through standard disks. Finally, we note that revealing the evolution of high redshift AGNs and heavily obscured "Compton-thick" AGNs are prime issues to be addressed by future X-ray observations.

\section{Acknowledgement}

I would like to thank all collaborators of mine that made it possible to conduct this research, including the colleagues who made enormous efforts in developing the Japanese X-ray observatories. I am grateful to the co-authors of the two key papers ${ }^{12), 53)}$ used in this review, in particular Masayuki Akiyama, the leading person in the optical identification programs of X-ray selected sources in the AMSS and SXDS projects.

\section{References}

1) Magorrian, J., Tremaine, S., Richstone, D., Bender, R., Bower, G., Dressler, A., Faber, S.M., Gebhardt, K., Green, R., Grillmair, C., Kormendy, J. and Lauer, T. (1998) The demography of massive dark objects in galaxy centers. Astron. J. 115, 2285-2305.

2) Ferrarese, L. and Merritt, D. (2000) A fundamental relation between supermassive black holes and their host galaxies. Astrophys. J. 539, L9-L12.

3) Gebhardt, K., Bender, R., Bower, G., Dressler, A., Faber, S.M., Filippenko, A.V., Green, R., Grillmair, C., Ho, L.C., Kormendy, J., Lauer, T.R., Magorrian, J., Pinkney, J., Richstone, D. and Tremaine, S. (2000) A relationship between nuclear black hole mass and galaxy velocity dispersion. Astrophys. J. 539, L13-L16.
4) Marconi, A. and Hunt, L.K. (2003) The relation between black hole mass, bulge mass, and nearinfrared luminosity. Astrophys. J. 589, L21-L24.

5) Häring, N. and Rix, H. (2004) On the black hole mass-bulge mass relation. Astrophys. J. 604, L89-L92.

6) Gültekin, K., Richstone, D.O., Gebhardt, K., Lauer, T.R., Tremaine, S., Aller, M.C., Bender, R., Dressler, A., Faber, S.M., Filippenko, A.V., Green, R., Ho, L.C., Kormendy, J., Magorrian, J., Pinkney, J. and Siopis, C. (2009) The $M-\sigma$ and $M-L$ relations in galactic bulges, and determinations of their intrinsic scatter. Astrophys. J. 698, 198-221.

7) Kormendy, J. and Ho, L.C. (2013) Coevolution (or not) of supermassive black holes and host galaxies. Annu. Rev. Astron. Astrophys. 51, 511-653.

8) Shakura, N.I. and Sunyaev, R.A. (1973) Black holes in binary systems. Observational appearance. Astron. Astrophys. 24, 337-355.

9) Fabian, A.C. (2012) Observational evidence of active galactic nuclei feedback. Annu. Rev. Astron. Astrophys. 50, 455-489.

10) Antonucci, R. (1993) Unified models for active galactic nuclei and quasars. Annu. Rev. Astron. Astrophys. 31, 473-521.

11) Awaki, H., Koyama, K., Inoue, H. and Halpern, J.P. (1991) X-ray implications of a unified model of Seyfert galaxies. Publ. Astron. Soc. Jpn. 43, 195212.

12) Ueda, Y., Akiyama, M., Hasinger, G., Miyaji, T. and Watson, M.G. (2014) Toward the standard population synthesis model of the X-ray background: Evolution of X-ray luminosity and absorption functions of active galactic nuclei including Compton-thick populations. Astrophys. J. 786, article id. 104.

13) Giacconi, R., Gursky, H., Paolini, F.R. and Rossi, B.B. (1962) Evidence for $\mathrm{x}$ rays from sources outside the solar system. Phys. Rev. Lett. 9, 439443.

14) Marshall, F.E., Boldt, E.A., Holt, S.S., Miller, R.B., Mushotzky, R.F., Rose, L.A., Rothschild, R.E. and Serlemitsos, P.J. (1980) The diffuse Xray background spectrum from 3 to $50 \mathrm{keV}$. Astrophys. J. 235, 4-10.

15) Mather, J.C., Cheng, E.S., Cottingham, D.A., Eplee, R.E., Jr., Fixsen, D.J., Hewagama, T., Isaacman, R.B., Jensen, K.A., Meyer, S.S., Noerdlinger, P.D., Read, S.M., Rosen, L.P., Shafer, R.A., Wright, E.L., Bennett, C.L., Boggess, N.W., Hauser, M.G., Kelsall, T., Moseley, S.H., Jr., Silverberg, R.F., Smoot, G.F., Weiss, R. and Wilkinson, D.T. (1994) Measurement of the cosmic microwave background spectrum by the COBE FIRAS instrument. Astrophys. J. 420, 439-444.

16) Piccinotti, G., Mushotzky, R.F., Boldt, E.A., Holt, S.S., Marshall, F.E., Serlemitsos, P.J. and Shafer, R.A. (1982) A complete X-ray sample of the high-latitude $\left(|b|>20^{\circ}\right)$ sky from HEAO $1 \mathrm{~A}-2$ : $\log N-\log S$ and luminosity functions. Astrophys. 
J. 253, 485-503.

17) Ueda, Y., Takahashi, T., Ishisaki, Y., Ohashi, T. and Makishima, K. (1999) The ASCA mediumsensitivity survey (The GIS catalog project) Source counts and evidence for emerging population of hard sources. Astrophys. J. 524, L11-L14.

18) Ueda, Y., Watson, M.G., Stewart, I.M., Akiyama, M., Schwope, A.D., Lamer, G., Ebrero, J., Carrera, F.J., Sekiguchi, K., Yamada, T., Simpson, C., Hasinger, G. and Mateos, S. (2008) The Subaru/XMM-Newton Deep Survey (SXDS). III. X-ray data. Astrophys. J. 179, 124-141.

19) Lehmer, B.D., Xue, Y.Q., Brandt, W.N., Alexander, D.M., Bauer, F.E., Brusa, M., Comastri, A., Gilli, R., Hornschemeier, A.E., Luo, B., Paolillo, M. Ptak, A., Shemmer, O., Schneider, D.P., Tozzi, P. and Vignali, C. (2012) The 4Ms Chandra Deep Field-South number counts apportioned by source class: Pervasive active galactic nuclei and the ascent of normal galaxies. Astrophys. J. 752, article id. 46.

20) Revnivtsev, M., Sazonov, S., Jahoda, K. and Gilfanov, M. (2004) RXTE all-sky slew survey. Catalog of X-ray sources at $|b|>10^{\circ}$. Astron. Astrophys. 418, 927-936.

21) Matsuoka, M., Kawasaki, K., Ueno, S., Tomida, H., Kohama, M., Suzuki, M., Adachi, Y., Ishikawa, M., Mihara, T., Sugizaki, M., Isobe, N., Nakagawa, Y., Tsunemi, H., Miyata, E., Kawai, N., Kataoka, J., Morii, M., Yoshida, A., Negoro, H., Nakajima, M., Ueda, Y., Chujo, H., Yamaoka, K., Yamazaki, O., Nakahira, S., You, T., Ishiwata, R., Miyoshi, S., Eguchi, S., Hiroi, K., Katayama, H. and Ebisawa, K. (2009) The MAXI mission on the ISS: Science and instruments for monitoring All-sky X-ray Images. Publ. Astron. Soc. Jpn. 61, 999-1010.

22) Hiroi, K., Ueda, Y., Isobe, N., Hayashida, M., Eguchi, S., Sugizaki, M., Kawai, N., Tsunemi, H., Matsuoka, M., Mihara, T., Yamaoka, K., Ishikawa, M., Kimura, M., Kitayama, H., Kohama, M., Matsumura, T., Morii, M., Nakagawa, Y.E., Nakahira, S., Nakajima, M., Negoro, H., Serino, M., Shidatsu, M., Sootome, T., Sugimori, K., Suwa, F., Toizumi, T., Tomida, H., Tsuboi, Y., Ueno, S., Usui, R., Yamamoto, T., Yamazaki, K. and Yoshida, A. (2011) The first MAXI/GSC catalog in the high galactic-latitude sky. Publ. Astron. Soc. Jpn. 63S, S677-S689.

23) Hiroi, K., Ueda, Y., Hayashida, M., Shidatsu, M., Sato, R., Kawamuro, T., Sugizaki, M., Nakahira, S., Serino, M., Kawai, N., Matsuoka, M., Mihara, T., Morii, M., Nakajima, M., Negoro, H., Sakamoto, T., Tomida, H., Tsuboi, Y., Tsunemi, H., Ueno, S., Yamaoka, K., Yoshida, A., Asada, M., Eguchi, S., Hanayama, T., Higa, M., Ishikawa, K., Ishikawa, M., Isobe, N., Kohama, M., Kimura, M., Morihana, K., Nakagawa, Y.E., Nakano, Y., Nishimura, Y., Ogawa, Y., Sasaki, M., Sugimoto, J., Takagi, T., Usui, R., Yamamoto, T., Yamauchi, M. and Yoshidome, K. (2013) The 37 month MAXI/GSC source catalog of the high galactic-latitude sky. Astrophys. J. Suppl. 207, article id. 36.

24) Markwardt, C.B., Tueller, J., Skinner, G.K., Gehrels, N., Barthelmy, S.D. and Mushotzky, R.F. (2005) The Swift/BAT high-latitude survey: First results. Astrophys. J. 633, L77-L80.

25) Beckmann, V., Gehrels, N., Shrader, C.R. and Soldi, S. (2006) The first INTEGRAL AGN catalog. Astrophys. J. 638, 642-652.

26) Voges, W., Aschenbach, B., Boller, Th., Bräuninger, H., Briel, U., Burkert, W., Dennerl, K., Englhauser, J., Gruber, R., Haberl, F., Hartner, G., Hasinger, G., Kürster, M., Pfeffermann, E., Pietsch, W., Predehl, P., Rosso, C., Schmitt, J.H.M.M., Trümper, J. and Zimmermann, H.U. (1999) The ROSAT all-sky survey bright source catalogue. Astron. Astrophys. 349, 389-405.

27) Ueda, Y., Eguchi, S., Terashima, Y., Mushotzky, R., Tueller, J., Markwardt, C., Gehrels, N., Hashimoto, Y. and Potter, S. (2007) Suzaku observations of active galactic nuclei detected in the Swift BAT survey: Discovery of a "new type" of buried supermassive black holes. Astrophys. J. 664, L79-L82.

28) Tanaka, Y., Inoue, H. and Holt, S.S. (1994) The X-ray astronomy satellite ASCA. Publ. Astron. Soc. Jpn. 46, L37-L41.

29) Ohashi, T., Ebisawa, K., Fukazawa, Y., Hiyoshi, K., Horii, M., Ikebe, Y., Ikeda, H., Inoue, H., Ishida, M., Ishisaki, Y., Ishizuka, T., Kamijo, S., Kaneda, H., Kohmura, Y., Makishima, K., Mihara, T., Tashiro, M., Murakami, T., Shoumura, R., Tanaka, Y., Ueda, Y., Taguchi, K., Tsuru, T. and Takeshima, T. (1996) The Gas Imaging Spectrometer on board ASCA. Publ. Astron. Soc. Jpn. 48, 157-170.

30) Serlemitsos, P.J., Jalota, L., Soong, Y., Kunieda, H., Tawara, Y., Tsusaka, Y., Suzuki, H., Sakima, Y., Yamazaki, T., Yoshioka, H., Furuzawa, A., Yamashita, K., Awaki, H., Itoh, M., Ogasaka, Y., Honda, H. and Uchibori, Y. (1995) The X-ray Telescope on board ASCA. Publ. Astron. Soc. Jpn. 47, 105-114.

31) Giacconi, R., Bechtold, J., Branduardi, G., Forman, W., Henry, J.P., Jones, C., Kellogg, E., van der Laan, H., Liller, W., Marshall, H., Murray, S.S., Pye, J., Schreier, E., Sargent, W.L.W., Seward, F. and Tananbaum, H. (1979) A high-sensitivity Xray survey using the Einstein Observatory and the discrete source contribution to the extragalactic X-ray background. Astrophys. J. 234, L1-L7.

32) Hasinger, G., Burg, R., Giacconi, R., Hartner, G., Schmidt, M., Trumper, J. and Zamorani, G. (1993) A deep X-ray survey in the Lockman-Hole and the soft X-ray $\log N-\log S$. Astron. Astrophys. 275, 1-15.

33) Hasinger, G., Burg, R., Giacconi, R., Schmidt, M., Trumper, J. and Zamorani, G. (1998) The ROSAT Deep Survey. I. X-ray sources in the Lockman Field. Astron. Astrophys. 329, 482-494.

34) Schmidt, M., Hasinger, G., Gunn, J., Schneider, D., Burg, R., Giacconi, R., Lehmann, I., MacKenty, 
J., Trumper, J. and Zamorani, G. (1998) The ROSAT deep survey. II. Optical identification, photometry and spectra of X-ray sources in the Lockman field. Astron. Astrophys. 329, 495-503.

35) Turner, T.J. and Pounds, K.A. (1989) The EXOSAT spectral survey of AGN. Mon. Not. R. Astron. Soc. 240, 833-880.

36) Williams, O.R., Turner, M.J.L., Stewart, G.C., Saxton, R.D., Ohashi, T., Makishima, K., Kii, T., Inoue, H., Makino, F., Hayashida, K. and Koyama, K. (1992) The X-ray spectra of highluminosity active galactic nuclei observed by Ginga. Astrophys. J. 389, 157-178.

37) Gendreau, K.C., Mushotzky, R., Fabian, A.C., Holt, S.S., Kii, T., Serlemitsos, P.J., Ogasaka, Y., Tanaka, Y., Bautz, M.W., Fukazawa, Y., Ishisaki, Y., Kohmura, Y., Makishima, K., Tashiro, M., Tsusaka, Y., Kunieda, H., Ricker, G.R. and Vanderspek, R.K. (1995) ASCA observations of the spectrum of the X-ray background. Publ. Astron. Soc. Jpn. 47, L5-L9.

38) Kushino, A., Ishisaki, Y., Morita, U., Yamasaki, N.Y., Ishida, M., Ohashi, T. and Ueda, Y. (2002) Study of the X-ray background spectrum and its large-scale fluctuation with ASCA. Publ. Astron. Soc. Jpn. 54, 327-352.

39) Ueda, Y., Takahashi, T., Inoue, H., Tsuru, T., Sakano, M., Ishisaki, Y., Ogasaka, Y., Makishima, K., Yamada, T., Ohta, K. and Akiyama, M. (1998) A population of faint galaxies that contribute to the cosmic X-ray background. Nature 391, 866-868.

40) Ueda, Y., Takahashi, T., Inoue, H., Tsuru, T. Sakano, M., Ishisaki, Y., Ogasaka, Y., Makishima, K., Yamada, T., Akiyama, M. and Ohta, K. (1999) $\log N-\log S$ relations and spectral properties of sources from the ASCA Large Sky Survey: Their implications for the origin of the cosmic X-ray background (CXB). Astrophys. J. 518, 656-671.

41) Akiyama, M., Ohta, K., Yamada, T., Kashikawa, N., Yagi, M., Kawasaki, W., Sakano, M., Tsuru, T., Ueda, Y., Takahashi, T., Lehmann, I., Hasinger, G. and Voges, W. (2000) Optical identification of the ASCA Large Sky Survey. Astrophys. J. 532, 700-727.

42) Ueda, Y., Ishisaki, Y., Takahashi, T., Makishima, K. and Ohashi, T. (2001) The ASCA Medium Sensitivity Survey (the GIS catalog project): Source catalog. Astrophys. J. Suppl. 133, 1-52.

43) Ueda, Y., Ishisaki, Y., Takahashi, T., Makishima, K. and Ohashi, T. (2005) The ASCA Medium Sensitivity Survey (The GIS catalog project): Source catalog II. Astrophys. J. Suppl. 161, $185-223$

44) Giommi, P., Perri, M. and Fiore, F. (2000) The BeppoSAX 2-10 keV survey. Astron. Astrophys. 362, 799-808.

45) Akiyama, M., Ueda, Y., Ohta, K., Takahashi, T. and Yamada, T. (2003) Optical identification of the ASCA Medium Sensitivity Survey in the northern sky: Nature of hard X-ray-selected luminous active galactic nuclei. Astrophys. J. Suppl. 148, 275-315.

46) Mushotzky, R.F., Cowie, L.L., Barger, A.J. and Arnaud, K.A. (2000) Resolving the extragalactic hard X-ray background. Nature 404, 459-464.

47) Brandt, W.N., Alexander, D.M., Hornschemeier, A.E., Garmire, G.P., Schneider, D.P., Barger, A.J., Bauer, F.E., Broos, P.S., Cowie, L.L., Townsley, L.K., Burrows, D.N., Chartas, G., Feigelson, E.D., Griffiths, R.E., Nousek, J.A. and Sargent, W.L.W. (2001) The Chandra Deep Field North survey. V. $1 \mathrm{Ms}$ source catalogs. Astron. J. 122, 2810-2832.

48) Giacconi, R., Rosati, P., Tozzi, P., Nonino, M., Hasinger, G., Norman, C., Bergeron, J., Borgani, S., Gilli, R., Gilmozzi, R. and Zheng, W. (2001) First results from the X-ray and optical survey of the Chandra Deep Field South. Astrophys. J. 551, 624-634.

49) Hasinger, G., Altieri, B., Arnaud, M., Barcons, X., Bergeron, J., Brunner, H., Dadina, M., Dennerl, K., Ferrando, P., Finoguenov, A., Griffiths, R.E., Hashimoto, Y., Jansen, F.A., Lumb, D.H., Mason, K.O., Mateos, S., McMahon, R.G., Miyaji, T., Paerels, F., Page, M.J., Ptak, A.F., Sasseen, T.P., Schartel, N., Szokoly, G.P., Trumper, J., Turner, M., Warwick, R.S. and Watson, M.G. (2001) XMM-Newton observation of the Lockman Hole. I. The X-ray data. Astron. Astrophys. 365, L45L50.

50) Nandra, K., Laird, E.S., Adelberger, K., Gardner, Jonathan, P., Mushotzky, R.F., Rhodes, J., Steidel, C.C., Teplitz, H.I. and Arnaud, K.A. (2005) A deep Chandra survey of the Groth Strip - I. The X-ray data. Mon. Not. R. Astron. Soc. 356, 568-586.

51) Hasinger, G., Cappelluti, N., Brunner, H., Brusa, M., Comastri, A., Elvis, M., Finoguenov, A., Fiore, F., Franceschini, A., Gilli, R., Griffiths, R.E., Lehmann, I., Mainieri, V., Matt, G., Matute, I., Miyaji, T., Molendi, S., Paltani, S., Sanders, D.B., Scoville, N., Tresse, L., Urry, C.M., Vettolani, P. and Zamorani, G. (2007) The XMMNewton wide-field survey in the COSMOS field. I. Survey description. Astrophys. J. Suppl. 172, 2937.

52) Brandt, W.N. and Hasinger, G. (2005) Deep extragalactic X-ray surveys. Annu. Rev. Astron. Astrophys. 43, 827-859.

53) Ueda, Y., Akiyama, M., Ohta, K. and Miyaji, T. (2003) Cosmological evolution of the hard X-ray active galactic nucleus luminosity function and the origin of the hard X-ray background. Astrophys. J. 598, 886-908.

54) Miyaji, T., Hasinger, G. and Schmidt, M. (2000) Soft X-ray AGN luminosity function from it ROSAT surveys. I. Cosmological evolution and contribution to the soft X-ray background. Astron. Astrophys. 353, 25-40.

55) Nobuta, K., Akiyama, M., Ueda, Y., Watson, M.G., Silverman, J., Hiroi, K., Ohta, K., Iwamuro, F., Yabe, K., Tamura, N., Moritani, Y., Sumiyoshi, 
M., Takato, N., Kimura, M., Maihara, T., Dalton, G., Lewis, I., Bonfield, D., Lee, H., Curtis-Lake, E., Macaulay, E., Clarke, F., Sekiguchi, K., Simpson, C., Croom, S., Ouchi, M., Hanami, H. and Yamada, T. (2012) Black hole mass and Eddington ratio distribution functions of X-rayselected broad-line AGNs at $z \sim 1.4$ in the Subaru/XMM-Newton Deep Field. Astrophys. J. $\mathbf{7 6 1}$, article id. 143.

56) Barger, A.J., Cowie, L.L., Mushotzky, R.F., Yang, Y., Wang, W.-H., Steffen, A.T. and Capak, P. (2005) The cosmic evolution of hard X-rayselected active galactic nuclei. Astron. J. 129, 578-609.

57) Hasinger, G., Miyaji, T. and Schmidt, M. (2005) Luminosity-dependent evolution of soft X-ray selected AGN. New Chandra and XMM-Newton surveys. Astron. Astrophys. 441, 417-434.

58) La Franca, F., Fiore, F., Comastri, A., Perola, G.C., Sacchi, N., Brusa, M., Cocchia, F., Feruglio, C., Matt, G., Vignali, C., Carangelo, N., Ciliegi, P., Lamastra, A., Maiolino, R., Mignoli, M., Molendi, S. and Puccetti, S. (2005) The HELLAS2XMM survey. VII. The hard X-ray luminosity function of AGNs up to $z=4$ : More absorbed AGNs at low luminosities and high redshifts. Astrophys. J. 635, 864-879.

59) Silverman, J.D., Green, P.J., Barkhouse, W.A., Kim, D.-W., Kim, M., Wilkes, B.J., Cameron, R.A., Hasinger, G., Jannuzi, B.T., Smith, M.G., Smith, P.S. and Tananbaum, H. (2008) The luminosity function of X-ray-selected active galactic nuclei: Evolution of supermassive black holes at high redshift. Astrophys. J. 679, 118-139.

60) Ebrero, J., Carrera, F.J., Page, M.J., Silverman, J.D., Barcons, X., Ceballos, M.T., Corral, A., Della Ceca, R. and Watson, M.G. (2009) The XMM-Newton serendipitous survey. VI. The Xray luminosity function. Astron. Astrophys. 493, $55-69$.

61) Yencho, B., Barger, A.J., Trouille, L. and Winter, L.M. (2009) The OPTX project. II. Hard X-ray luminosity functions of active galactic nuclei for $z \lesssim 5$. Astrophys. J. 698, 380-396.

62) Aird, J., Nandra, K., Laird, E.S., Georgakakis, A., Ashby, M.L.N., Barmby, P., Coil, A.L., Huang, J.-S., Koekemoer, A.M., Steidel, C.C. and Willmer, C.N.A. (2010) The evolution of the hard X-ray luminosity function of AGN. Mon. Not. R. Astron. Soc. 401, 2531-2551.

63) Miyaji, T., Hasinger, G., Salvato, M., Brusa, M., Cappelluti, N., Civano, F., Puccetti, S., Elvis, M., Brunner, H., Fotopoulou, S., Ueda, Y., Griffiths, R.E., Koekemoer, A.M., Akiyama, M., Comastri, A., Gilli, R., Lanzuisi, G., Merloni, A. and Vignali, C. (2015) Detailed shape and evolutionary behavior of the X-ray luminosity function of active galactic nuclei. Astrophys. J. (in press) (arXiv:1503.00056).

64) Lawrence, A. and Elvis, M. (1982) Obscuration and the various kinds of Seyfert galaxies. Astrophys. J. 256, 410-426.
65) Simpson, C. (2005) The luminosity dependence of the type 1 active galactic nucleus fraction. Mon. Not. R. Astron. Soc. 360, 565-572.

66) Maiolino, R., Shemmer, O., Imanishi, M., Netzer, H., Oliva, E., Lutz, D. and Sturm, E. (2007) Dust covering factor, silicate emission, and star formation in luminous QSOs. Astron. Astrophys. 468, 979-992.

67) Toba, Y., Oyabu, S., Matsuhara, H., Malkan, M.A., Gandhi, P., Nakagawa, T., Isobe, N., Shirahata, M., Oi, N., Ohyama, Y., Takita, S., Yamauchi, C. and Yano, K. (2014) Luminosity and redshift dependence of the covering factor of active galactic nuclei viewed with WISE and Sloan Digital Sky Survey. Astrophys. J. 788, article id. 45.

68) Ricci, C., Paltani, S., Awaki, H., Petrucci, P.-O., Ueda, Y. and Brightman, M. (2013) Luminositydependent unification of active galactic nuclei and the X-ray Baldwin effect. Astron. Astrophys. 553, id.A29.

69) Iwasawa, K. and Taniguchi, Y. (1993) The X-ray Baldwin effect. Astrophys. J. 413, L15-L18.

70) Kishimoto, M., Hönig, S.F., Antonucci, R., Millour, F., Tristram, K.R.W. and Weigelt, G. (2011) Mapping the radial structure of AGN tori. Astron. Astrophys. 536, id.A78.

71) Ballantyne, D.R., Everett, J.E. and Murray, N. (2006) Connecting galaxy evolution, star formation, and the cosmic X-ray background. Astrophys. J. 639, 740-752.

72) Treister, E. and Urry, C.M. (2006) The evolution of obscuration in active galactic nuclei. Astrophys. J. 652, L79-L82.

73) Hasinger, G. (2008) Absorption properties and evolution of active galactic nuclei. Astron. Astrophys. 490, 905-922.

74) Page, M.J., Stevens, J.A., Ivison, R.J. and Carrera, F.J. (2004) The evolutionary sequence of active galactic nuclei and galaxy formation revealed. Astrophys. J. 611, L85-L88.

75) Comastri, A., Setti, G., Zamorani, G. and Hasinger, G. (1995) The contribution of AGNs to the X-ray background. Astron. Astrophys. 296, 1-12.

76) Gilli, R., Comastri, A. and Hasinger, G. (2007) The synthesis of the cosmic X-ray background in the Chandra and XMM-Newton era. Astron. Astrophys. 463, 79-96.

77) Treister, E., Urry, C.M. and Virani, S. (2009) The space density of Compton-thick active galactic nucleus and the X-ray background. Astrophys. J. 696, 110-120.

78) Ajello, M., Greiner, J., Sato, G., Willis, D.R., Kanbach, G., Strong, A.W., Diehl, R., Hasinger, G., Gehrels, N., Markwardt, C.B. and Tueller, J. (2008) Cosmic X-ray background and earth albedo spectra with Swift BAT. Astrophys. J. 689, 666-677.

79) Soltan, A. (1982) Masses of quasars. Mon. Not. R. Astron. Soc. 200, 115-122.

80) Ichimaru, S. (1977) Bimodal behavior of accretion disks - Theory and application to Cygnus X-1 
transitions. Astrophys. J. 214, 840-855.

81) Hopkins, P.F., Richards, G.T. and Hernquist, L. (2007) An observational determination of the bolometric quasar luminosity function. Astrophys. J. 654, 731-753.

82) Marconi, A., Risaliti, G., Gilli, R., Hunt, L.K. Maiolino, R. and Salvati, M. (2004) Local supermassive black holes, relics of active galactic nuclei and the X-ray background. Mon. Not. R. Astron. Soc. 351, 169-185.

83) Yu, Q. and Tremeine, S. (2002) Observational constraints on growth of massive black holes. Mon. Not. R. Astron. Soc. 335, 965-976.

84) Shankar, F., Salucci, P., Granato, G.L., De Zotti, G. and Danese, L. (2004) Supermassive black hole demography: the match between the local and accreted mass functions. Mon. Not. R. Astron. Soc. 354, 1020-1030.

85) Cao, X.-W. and Li, F. (2008) Rapidly spinning massive black holes in active galactic nuclei: evidence from the black hole mass function. Mon. Not. R. Astron. Soc. 390, 561-566.

86) Madau, P. and Dickinson, M. (2015) Cosmic starformation history. Annu. Rev. Astron. Astrophys. 52, 415-486.

87) Boyle, B.J. and Terlevich, R.J. (2015) The cosmological evolution of the QSO luminosity density and of the star formation rate. Mon. Not. R. Astron. Soc. 293, L49-L51.

88) Cowie, L.L., Songaila, A., Hu, E.M. and Cohen, J.G. (1996) New insight on galaxy formation and evolution from keck spectroscopy of the Hawaii deep fields. Astron. J. 112, 839-864.

89) Kodama, T., Yamada, T., Akiyama, M., Aoki, K., Doi, M., Furusawa, H., Fuse, T., Imanishi, M., Ishida, C., Iye, M., Kajisawa, M., Karoji, H., Kobayashi, N., Komiyama, Y., Kosugi, G., Maeda, Y., Miyazaki, S., Mizumoto, Y., Morokuma, T., Nakata, F., Noumaru, J., Ogasawara, R., Ouchi, M., Sasaki, T., Sekiguchi, K., Shimasaku, K., Simpson, C., Takata, T., Tanaka, I., Ueda, Y., Yasuda, N. and Yoshida, M. (2004) Down-sizing in galaxy formation at $z \sim 1$ in the Subaru/ XMM-Newton Deep Survey (SXDS). Mon. Not. R. Astron. Soc. 350, 1005-1014.

90) Fontanot, F., Monaco, P., Cristiani, S. and Tozzi, P. (2006) The effect of stellar feedback and quasar winds on the active galactic nucleus population. Mon. Not. R. Astron. Soc. 373, 1173-1187.

91) Kauffmann, G. and Haehnelt, M. (2000) A unified model for the evolution of galaxies and quasars. Mon. Not. R. Astron. Soc. 311, 576-588.

92) Wyithe, J.S.B. and Loeb, A. (2003) Self-regulated growth of supermassive black holes in galaxies as the origin of the optical and X-ray luminosity functions of quasars. Astrophys. J. 595, 614-623.

93) Marulli, F., Bonoli, S., Branchini, E., Moscardini, L. and Springel, V. (2008) Modelling the cosmological co-evolution of supermassive black holes and galaxies - I. BH scaling relations and the AGN luminosity function. Mon. Not. R. Astron. Soc. 385, 1846-1858.
94) Fanidakis, N., Baugh, C.M., Benson, A.J., Bower, R.G., Cole, S., Done, C., Frenk, C.S., Hickox, R.C., Lacey, C. and Lagos, C. del P. (2012) The evolution of active galactic nuclei across cosmic time: what is downsizing? Mon. Not. R. Astron. Soc. 419, 2797-2820.

95) Hirschmann, M., Somerville, R.S., Naab, T. and Burkert, A. (2012) Origin of the antihierarchical growth of black holes. Mon. Not. R. Astron. Soc. 426, 237-257.

96) Hirschmann, M., Dolag, K., Saro, A., Bachmann, L., Borgani, S. and Burkert, A. (2014) Cosmological simulations of black hole growth: AGN luminosities and downsizing. Mon. Not. R. Astron. Soc. 442, 2304-2324.

97) Enoki, M., Ishiyama, T., Kobayashi, M.A.R. and Nagashima, M. (2014) Anti-hierarchical evolution of the active galactic nucleus space density in a hierarchical universe. Astrophys. J. 794, article id. 69.

98) Kiuchi, G., Ohta, K., Akiyama, M., Aoki, K. and Ueda, Y. (2006) Host galaxies of hard X-rayselected type 2 active galactic nuclei at intermediate redshifts. Astrophys. J. 647, 892-900.

99) Georgakakis, A., Coil, A.L., Laird, E.S., Griffith, R.L., Nandra, K., Lotz, J.M., Pierce, C.M., Cooper, M.C., Newman, J.A. and Koekemoer, A.M. (2009) Host galaxy morphologies of X-ray selected AGN: assessing the significance of different black hole fuelling mechanisms to the accretion density of the Universe at $z \sim 1$. Mon. Not. R. Astron. Soc. 397, 623-633.

100) Mortlock, D.J., Warren, S.J., Venemans, B.P., Patel, M., Hewett, P.C., McMahon, R.G., Simpson, C., Theuns, T., Gonzáles-Solares, E.A., Adamson, A., Dye, S., Hambly, N.C., Hirst, P., Irwin, M.J., Kuiper, E., Lawrence, A. and Röttgering, H.J.A. (2011) A luminous quasar at a redshift of $z=7.085$. Nature 474, 616-619.

101) Akylas, A., Georgakakis, A., Georgantopoulos, I., Brightman, M. and Nandra, K. (2012) Constraining the fraction of Compton-thick AGN in the Universe by modelling the diffuse X-ray background spectrum. Astron. Astrophys. 546, id.A98.

102) Sanders, D.B., Soifer, B.T., Elias, J.H., Madore, B.F., Matthews, K., Neugebauer, G. and Scoville, N.Z. (1988) Ultraluminous infrared galaxies and the origin of quasars. Astrophys. J. 325, 74-91.

103) Hopkins, P.F., Hernquist, L., Cox, T.J., Di Matteo, T., Robertson, B. and Springel, V. (2006) A unified, merger-driven model of the origin of starbursts, quasars, the cosmic X-ray background, supermassive black holes, and galaxy spheroids. Astrophys. J. Suppl. 163, 1-49.

104) Imanishi, M., Dudley, C.C., Maiolino, R., Maloney, P.R., Nakagawa, T. and Risaliti, G. (2007) A spitzer IRS low-resolution spectroscopic search for buried AGNs in nearby ultraluminous infrared galaxies: A constraint on geometry between energy sources and dust. Astrophys. J. Suppl. 171, 72-100.

105) Alexander, D.M., Stern, D., Del Moro, A., 
Lansbury, G.B., Assef, R.J., Aird, J., Ajello, M., Ballantyne, D.R., Bauer, F.E., Boggs, S.E., Brandt, W.N., Christensen, F.E., Civano, F., Comastri, A., Craig, W.W., Elvis, M., Grefenstette, B.W., Hailey, C.J., Harrison, F.A., Hickox, R.C., Luo, B., Madsen, K.K., Mullaney, J.R., Perri, M., Puccetti, S., Saez, C., Treister, E., Urry, C.M., Zhang, W.W., Bridge, C.R.,
Eisenhardt, P.R.M., Gonzalez, A.H., Miller, S.H. and Tsai, C.W. (2013) The NuSTAR extragalactic survey: A first sensitive look at the high-energy cosmic X-ray background population. Astrophys. J. 773, article id. 125.

(Received Feb. 28, 2015; accepted Apr. 9, 2015)

\section{Profile}

Yoshihiro Ueda was born in Hyogo prefecture, Japan in 1969. He graduated from the Department of Physics, Faculty of Science, the University of Tokyo in 1991 and received his Ph.D. degree from the same university in 1996. From 1996 he worked as research associate at the Institute of Space and Astronautical Science (ISAS), Ministry of Education, Science and Culture, which became a part of the Japan Aerospace eXploration Agency (JAXA) in 2003. In 2005, he moved to Department of Astronomy, Graduate School of Science, Kyoto University as associate professor. He has been deeply involved in Japanese X-ray astronomical missions including ASCA, Suzaku, and Monitor of All-sky X-ray Image (MAXI). His main research theme is observational studies of black holes over a wide range of masses based on X-ray/multi-wavelength astronomy. He

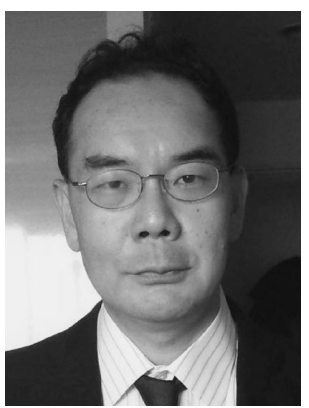
was awarded the 15th Young Scientist Award of the Astronomical Society of Japan in 2004, and the 30th Inoue Prize for Science in 2014. 